\title{
Associations of common IL-4 gene polymorphisms with cancer risk: A meta-analysis
}

\author{
YINGXIAN JIA ${ }^{1,2}$, XIAOCHUAN XIE ${ }^{3 *}$, XIAOHAN SHI ${ }^{1,2^{*}}$ and SHANGWEI LI ${ }^{1,2}$ \\ ${ }^{1}$ Division of Reproductive Medical Center, and ${ }^{2}$ Key Laboratory of Birth Defects and Related Diseases \\ of Women and Children, West China Second University Hospital; ${ }^{3}$ Department of Cardiology, \\ West China Hospital, Sichuan University, Chengdu, Sichuan 610041, P.R. China
}

Received June 2, 2016; Accepted April 7, 2017

DOI: $10.3892 / \mathrm{mmr} .2017 .6822$

\begin{abstract}
Cancer incidence is dramatically increasing worldwide, therefore improved prediction and therapeutic methods are needed. Single nucleotide polymorphisms in cytokine genes may contribute to carcinogenesis. Interleukin (IL)-4 gene polymorphisms have been intensively studied with regard to their associations with cancer. However, the results of these previous studies remain inconclusive. The present study, therefore, aimed to conduct a meta-analysis of previously published studies in order to clarify the association of IL-4 with cancer risk. Eligible published articles were searched in Medline, PubMed, Embase and China National Knowledge Infrastructure databases up to March 2016. Odds ratios and $95 \%$ confidence intervals were used to identify potential associations between IL-4 genetic polymorphisms and the risk of cancer. A meta-analysis was then performed on 10,873 patients and 14,328 controls for IL-4 rs2243250 polymorphism, 3,970 patients and 5,686 controls for IL-4 rs2070874 polymorphism, and 1,896 patients and 2,526 controls for IL-4 rs79071878 polymorphism. A significant association with cancer risk was observed for rs2243250 and rs79071878 polymorphisms. In the subgroup analysis by cancer type, rs2243250 polymorphism was demonstrated to be associated with an increased risk of gastric cancer and breast cancer, rs2070874 polymorphism was correlated with leukemia and oral carcinoma, and rs79071878 polymorphism was relevant to bladder carcinoma risk. In the subgroup analysis by ethnicity, IL-4 rs2243250 polymorphism was demonstrated to be associated with cancer risk in both Caucasian and Asian populations,
\end{abstract}

Correspondence to: Professor Shangwei Li, Division of Reproductive Medical Center, West China Second University Hospital, Sichuan University, 20, 3rd Section of Renming South Road, Chengdu, Sichuan 610041, P.R. China

E-mail: lishangwei1125@163.com

*Contributed equally

Key words: interleukin-4, polymorphisms, cancer, meta-analysis rs2070874 was associated with cancer risk in Asian populations, while rs79071878 polymorphism was associated with cancer risk in Caucasian populations. In conclusion, the present results suggested that the IL-4 rs2243250 and rs79071878 polymorphisms were associated with cancer susceptibility. Further subgroup analyses revealed that the effects of IL-4 gene polymorphisms on cancer risk may vary by cancer type and by ethnicity.

\section{Introduction}

It was estimated that there were $\sim 14$ million new cancer cases in 2012 and the number is expected to rise to 22 million in the next two decades (1). Cancer-associated mortality, meanwhile, was $\sim 8.2$ million in 2012 and is predicted to rise to 13 million by 2032 (1). Thus, an improved understanding of the pathogenic mechanisms of cancer is of great importance. At present, it is widely accepted that cancer is a multifactorial and complex disease resulting from interaction between environmental and genetic factors (2).

Single nucleotide polymorphisms (SNPs) are frequently occurring variations in the human genome, and have been extensively investigated in genetic studies of cancer. Recent studies have demonstrated that SNPs of multiple genes may have an important role in cancer occurrence and progression (3). In addition, numerous publications have reported that cytokine gene polymorphisms may affect inflammatory-related pathways, and influence susceptibility to different types of cancer $(4,5)$. Interleukin-4 (IL-4) is a potent regulator of antitumor immune responses with both tumor-promoting and tumor-inhibiting properties, since it has both immunosuppressive and anti-angiogenic functions (6-9). Consequently, certain genetic polymorphisms of IL-4 gene are considered as good candidates for cancer susceptibility prediction. To date, several studies have aimed to assess the potential association of IL-4 polymorphisms rs 2243250 [-590C to T, 5'untranslated region (UTR)], rs2070874 (-34C to T, 5' UTR) and rs79071878 (intron-3, $70 \mathrm{bp}$ variable number tandem repeat, VNTR) with cancer risk, but the results remain inconsistent. Therefore, a meta-analysis was performed in the present study in order to better elucidate the roles of IL-4 gene polymorphisms in the occurrence and progression of cancer. 


\section{Materials and methods}

Study identification and selection. Potentially relevant articles were independently identified by three investigators from the Medline (http://www.medline.com/), PubMed (https://www. ncbi.nlm.nih.gov/pubmed), Embase (https://www.embase. com) and China National Knowledge Infrastructure databases (http://www.cnki.net/). The searching terms were as follows: (Interleukin-4 OR IL-4 OR Interleukin 4 OR IL 4) AND (polymorphism OR variant OR genotype OR allele) AND (cancer OR tumor OR carcinoma OR neoplasm). In addition, the reference lists of retrieved articles were searched manually for additional eligible studies. Among studies with overlapping data published by the same authors, only the most recent and complete study was included in the present meta-analysis.

Inclusion and exclusion criteria. The following inclusion criteria were used to select eligible articles: i) Case-control study of cancer cases and healthy controls; ii) investigate the relationship between IL-4 gene polymorphisms and cancer risk; iii) provide both genotype and allele distributions inpatients and controls; iv) full text in English or Chinese available. Articles were excluded if: i) The study was duplicated; ii) the analyses were based on linkage considerations; iii) the report was not original (reviews or meta-analyses).

Data extraction and quality assessment. The following information was extracted from all included studies independently by two authors: i) Name of the first author; ii) year of publication; iii) country in which the study was conducted; iv) ethnicity of study population; v) cancer type; vi) allele and genotype frequencies of IL-4 gene polymorphisms in cases and controls; vii) P-value of Hardy-Weinberg equilibrium (HWE) in the control group. The Newcastle-Ottawa quality assessment scale was used to evaluate the quality of all included studies (10). This rating scale has a score range of 0 to 9 , and studies with scores $>7$ were assumed to be of high quality. Two reviewers performed data extraction and quality assessment independently. When necessary, the reviewers wrote to the corresponding authors for extra information or raw data. Disagreements between reviewers were resolved by discussion until a consensus was achieved. The final results were reviewed by a senior reviewer.

Statistical analysis. All statistical analyses were performed with Review Manage version 5.3 (Cochrane, London, United Kingdom). HWE in the control group was estimated using the $\chi^{2}$ test. Odds ratios (ORs) and $95 \%$ confidence intervals (CIs) were used to evaluate the strength of the associations between IL-4 gene polymorphisms and cancer susceptibility. In addition, heterogeneity among studies was assessed using the $\mathrm{Q}$ test and $\mathrm{I}^{2}$ statistics. When the probability value (P-value) of $\mathrm{Q}$ test was $<0.1$ or $\mathrm{I}^{2}$ was $>50 \%$, inter-study heterogeneity was considered to be significant, and the random-effects model (REM) was employed for analyses. Otherwise, the fixed-effect model (FEM) was applied for analyses. First, associations based on all study subjects were analyzed, and then subgroup analyses by cancer type and ethnicity were performed to obtain the cancer type-specific effects and the ethnic-specific effects of IL-4 polymorphisms. Sensitivity analyses were conducted by sequentially omitting one individual study each time to assess the stability of the results. Furthermore, the possible publication bias was evaluated by using funnel plots (data not shown).

\section{Results}

Characteristics of eligible studies. The literature search identified 1,237 eligible articles. After reading titles and abstracts, a total of 94 articles were selected for further evaluation. Amongst these, 51 articles were excluded based on the inclusion and exclusion criteria, as described in the Methods. Finally, 43 articles (11-53), 33 studies focusing on polymorphism rs2243250, 11 studies onrs2070874, and 10 studies on rs79071878, were included in the meta-analysis. The majority of the articles were published in English, except for three that were published in Chinese. A schematic of the selection process is illustrated in Fig. 1.

IL-4 rs2243250 polymorphism and the risk of cancer. For IL-4 rs2243250 polymorphism, a total of 33 studies including 10,873 cancer cases and 14,328 normal controls were investigated. Deviations from HWE were observed in 9 studies, while the other 24 studies were in accordance with HWE (Table I). As illustrated in Fig. 2, the meta-analysis identified a significant association between IL-4 rs2243250 polymorphism and cancer risk (CT vs. CC/TT: $\mathrm{P}=0.008$, $\mathrm{OR}=0.88,95 \%$ CI $0.80-0.97$ ) with an overt heterogeneity across studies $\left(I^{2}=56 \%\right)$. Subgroup analyses were then performed based on cancer type (Table II). The results suggested that the IL-4 rs2243250 polymorphism was significantly associated with an increased risk of gastric cancer (CT vs. TT: $\mathrm{P}=0.004, \mathrm{OR}=0.75,95 \%$ CI $0.61-0.91$; $\mathrm{CT}$ vs. $\mathrm{CC} / \mathrm{TT}$ : $\mathrm{P}=0.002, \mathrm{OR}=0.77,95 \% \mathrm{CI} 0.66-0.91$; and $\mathrm{C}$ vs. T: $\mathrm{P}=0.04, \mathrm{OR}=1.15,95 \% \mathrm{CI} 1.01-1.32$ ), breast cancer (CC vs. CT: $\mathrm{P}=0.05, \mathrm{OR}=1.21,95 \%$ CI $1.00-1.46$; TT vs. CC: $\mathrm{P}=0.04, \mathrm{OR}=0.56,95 \%$ CI $0.33-0.97$; $\mathrm{CC}$ vs. CT/TT: $\mathrm{P}=0.02, \mathrm{OR}=1.25,95 \%$ CI $1.04-1.51$; and $\mathrm{C}$ vs. $\mathrm{T}: \mathrm{P}=0.007$, $\mathrm{OR}=1.25,95 \%$ CI 1.06-1.47), lung cancer (CT vs. CC/TT: $\mathrm{P}=0.02, \mathrm{OR}=0.84,95 \%$ CI $0.75-0.97$ ), prostate cancer (CT vs. TT: $\mathrm{P}=0.004, \mathrm{OR}=1.48,95 \%$ CI $1.14-1.92$;T vs. $\mathrm{CC}$ : $\mathrm{P}=0.0009, \mathrm{OR}=0.48,95 \% \mathrm{CI} 0.31-0.74 ; \mathrm{CT}$ vs. $\mathrm{CC} / \mathrm{TT}$ : $\mathrm{P}=0.02, \mathrm{OR}=1.33,95 \%$ CI $1.05-1.69$; and TT vs. $\mathrm{CC} / \mathrm{CT}$ : $\mathrm{P}=0.0004, \mathrm{OR}=0.64,95 \%$ CI $0.50-0.82)$ and leukemia $(\mathrm{CC}$ vs. CT: $\mathrm{P}=0.005, \mathrm{OR}=5.35,95 \% \mathrm{CI} 1.64-17.47 ; \mathrm{CC}$ vs. $\mathrm{CT} / \mathrm{TT}$ : $\mathrm{P}=0.01, \mathrm{OR}=4.67,95 \%$ CI 1.42-15.31; and $\mathrm{CT}$ vs. $\mathrm{CC} / \mathrm{TT}$ : $\mathrm{P}=0.005, \mathrm{OR}=0.19,95 \%$ CI 0.06-0.61). Studies in each cancer subgroup were homogenous. No significant association between IL-4 rs 2243250 polymorphism and cancer risk was identified for oral carcinoma, colorectal cancer, skin cancer, hepatocellular carcinoma, lymphoma, bladder cancer, brain tumor, testicular tumor, renal cell carcinoma, and brain tumor (Table II). Subgroup analyses were also conducted by ethnicity. As illustrated in Table II, a significant association between IL-4 rs2243250 polymorphism and cancer risk was identified in both Caucasian (CT vs. TT: $\mathrm{P}=0.03, \mathrm{OR}=0.82$, 95\% CI 0.68-0.98, $\mathrm{I}^{2}=46 \%$; CT vs. CC/TT: $\mathrm{P}=0.02, \mathrm{OR}=0.79$, 95\% CI 0.66-0.96, $\mathrm{I}^{2}=64 \%$ ) and Asian populations (CT vs. CC/TT: $\mathrm{P}=0.006, \mathrm{OR}=0.89,95 \%$ CI $0.82-0.97, \mathrm{I}^{2}=36 \%$ ). 


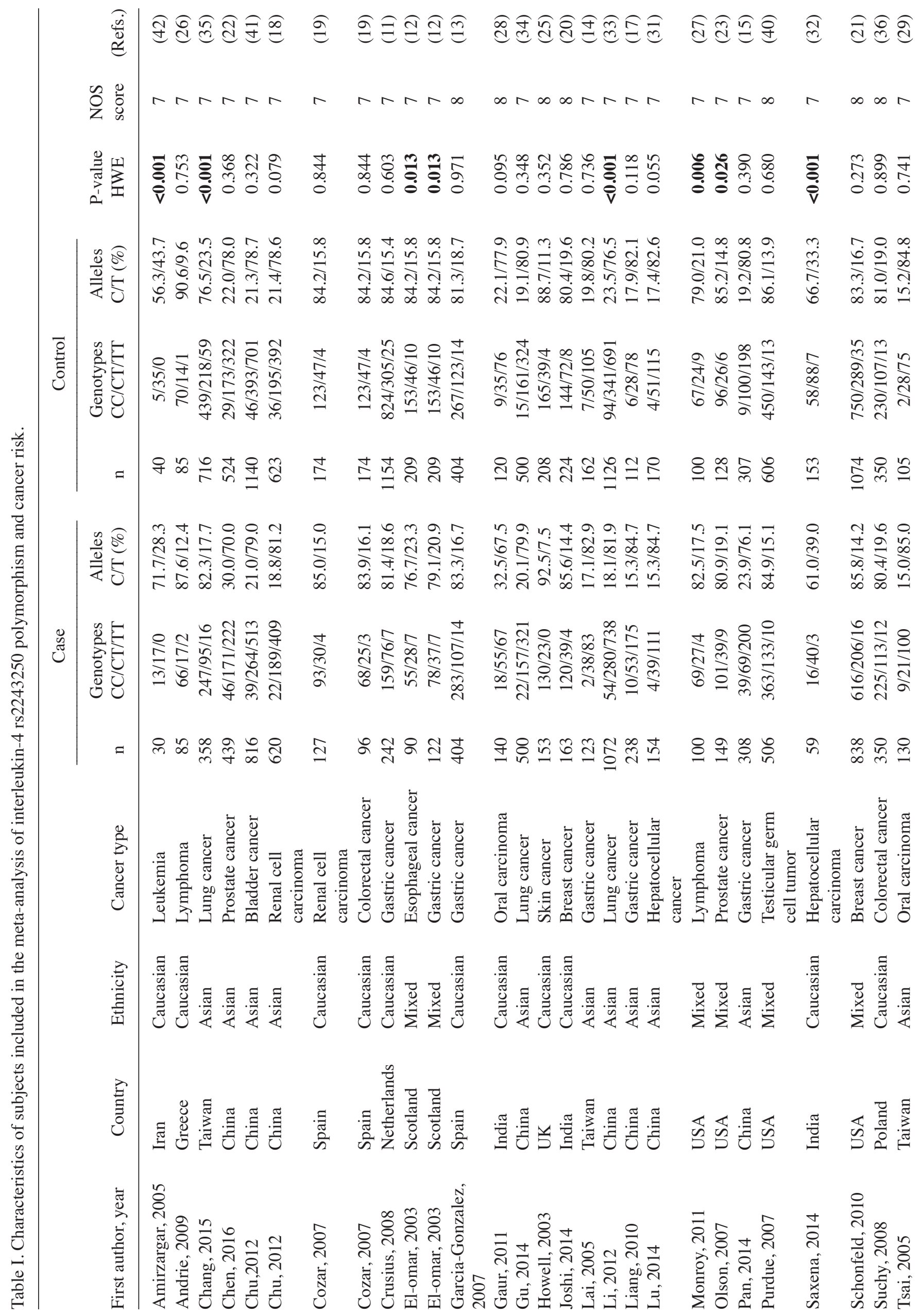




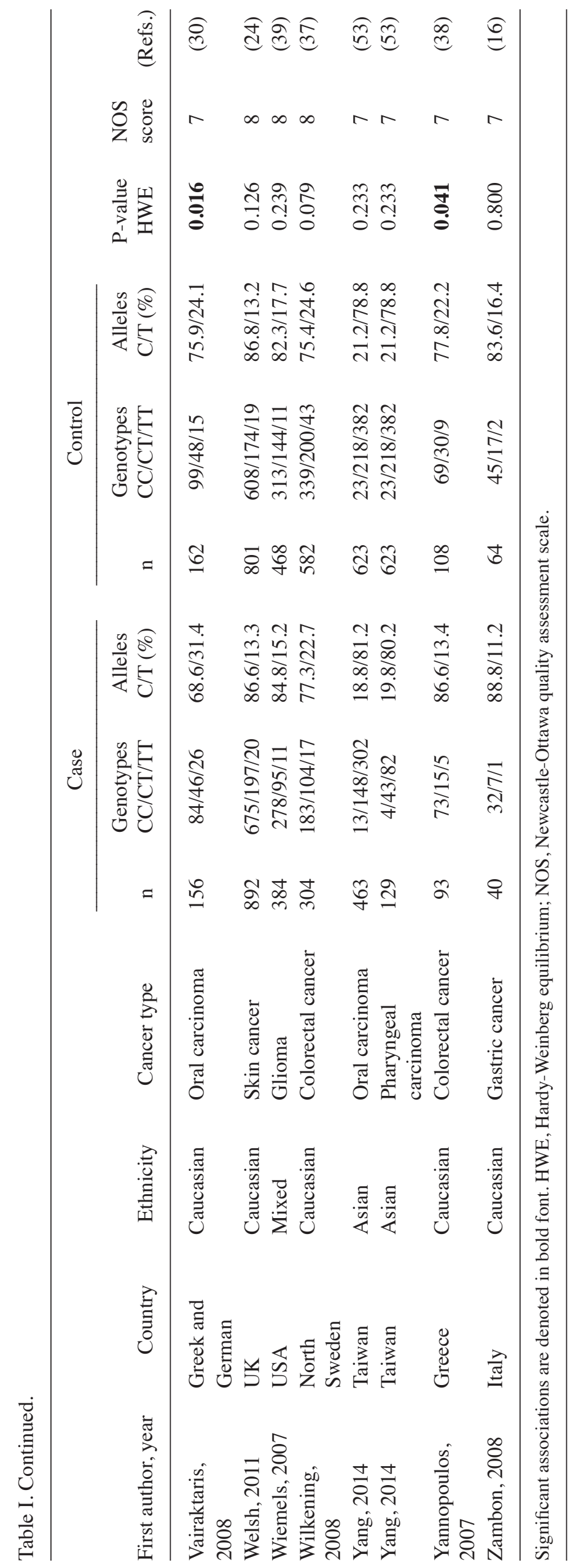


Table II. Subgroup analyses for interleukin-4 rs2243250 polymorphism and cancer risk.

A, Gastric cancer $\left(n=6^{a}\right)$

\begin{tabular}{|c|c|c|c|c|}
\hline Variable & P-value & OR $(95 \% \mathrm{Cl})$ & I-square $(\%)$ & $\begin{array}{c}\text { P-value } \\
\text { for the } \\
\text { heterogeneity }\end{array}$ \\
\hline CC vs. CT & 0.47 & $1.24(0.69-2.20)$ & $78 \%$ & 0.0003 \\
\hline CT vs. TT & 0.004 & $0.75(0.61-0.91)$ & $0 \%$ & 0.85 \\
\hline TT vs. CC & 0.57 & $0.81(0.40-1.66)$ & $63 \%$ & 0.02 \\
\hline $\mathrm{CC}$ vs. $\mathrm{CT}+\mathrm{TT}$ & 0.11 & $1.42(0.92-2.20)$ & $68 \%$ & 0.007 \\
\hline $\mathrm{CT}$ vs. $\mathrm{CC}+\mathrm{TT}$ & 0.002 & 0.77 (0.66-0.91) & $0 \%$ & 0.62 \\
\hline $\mathrm{TT}$ vs. $\mathrm{CC}+\mathrm{CT}$ & 0.63 & $1.06(0.85-1.32)$ & $0 \%$ & 0.95 \\
\hline C vs. T & 0.04 & 1.15 (1.01-1.32) & $21 \%$ & 0.28 \\
\hline \multicolumn{5}{|c|}{$B$, Oral carcinoma $\left(n=3^{a}\right)$} \\
\hline $\mathrm{CC}$ vs. CT & 0.43 & $1.41(0.60-3.30)$ & $45 \%$ & 0.14 \\
\hline CT vs. TT & 0.68 & $0.84(0.37-1.91)$ & $80 \%$ & 0.007 \\
\hline TT vs. CC & 0.67 & $0.78(0.25-2.44)$ & $77 \%$ & 0.01 \\
\hline $\mathrm{CC}$ vs. $\mathrm{CT}+\mathrm{TT}$ & 0.45 & $0.41(0.58-3.47)$ & $70 \%$ & 0.04 \\
\hline $\mathrm{CT}$ vs. $\mathrm{CC}+\mathrm{TT}$ & 0.90 & $0.96(0.54-1.71)$ & $70 \%$ & 0.03 \\
\hline TT vs. $\mathrm{CC}+\mathrm{CT}$ & 0.83 & $1.09(0.50-2.39)$ & $82 \%$ & 0.004 \\
\hline C vs. T & 0.88 & $1.05(0.60-1.84)$ & $82 \%$ & 0.004 \\
\hline \multicolumn{5}{|c|}{$C$, Colorectal cancer $\left(n=3^{a}\right)$} \\
\hline CC vs. TT & 0.51 & $1.12(0.80-1.57)$ & $55 \%$ & 0.11 \\
\hline CT vs. TT & 0.43 & $1.20(0.76-1.90)$ & $0 \%$ & 0.86 \\
\hline TT vs. CC & 0.83 & $1.04(0.70-1.55)$ & $0 \%$ & 0.41 \\
\hline $\mathrm{CC}$ vs. $\mathrm{CT}+\mathrm{TT}$ & 0.39 & $1.16(0.83-1.62)$ & $58 \%$ & 0.09 \\
\hline $\mathrm{CT}$ vs. $\mathrm{CC}+\mathrm{TT}$ & 0.74 & $0.97(0.79-1.19)$ & $50 \%$ & 0.14 \\
\hline TT vs. $\mathrm{CC}+\mathrm{CT}$ & 0.23 & $0.77(0.50-1.19)$ & $0 \%$ & 0.84 \\
\hline C vs. T & 0.31 & $1.15(0.88-1.52)$ & $56 \%$ & 0.10 \\
\hline
\end{tabular}

$\mathrm{D}$, Lung cancer $\left(\mathrm{n}=3^{\mathrm{a}}\right)$

\begin{tabular}{|c|c|c|c|c|}
\hline CC vs. CT & 0.97 & $0.99(0.67-1.47)$ & $63 \%$ & 0.07 \\
\hline CT vs. TT & 0.19 & $0.85(0.67-1.08)$ & $54 \%$ & 0.14 \\
\hline TT vs. CC & 0.75 & $0.87(0.35-2.17)$ & $89 \%$ & 0.00001 \\
\hline $\mathrm{CC}$ vs. $\mathrm{CT}+\mathrm{TT}$ & 0.89 & $1.05(0.54-2.01)$ & $88 \%$ & 0.0002 \\
\hline $\mathrm{CT}$ vs. $\mathrm{CC}+\mathrm{TT}$ & 0.02 & $0.84(0.75-0.97)$ & $0 \%$ & 0.58 \\
\hline TT vs. $\mathrm{CC}+\mathrm{CT}$ & 0.86 & $0.96(0.62-1.49)$ & $85 \%$ & 0.001 \\
\hline C vs. T & 0.92 & $1.02(0.68-1.54)$ & $92 \%$ & 0.00001 \\
\hline
\end{tabular}

E, Skin cancer $\left(n=2^{a}\right)$

\begin{tabular}{lllrr}
\hline CC vs. CT & 0.59 & $1.06(0.86-1.31)$ & $0 \%$ & 0.39 \\
CT vs. TT & 0.84 & $1.07(0.57-2.00)$ & $23 \%$ & 0.25 \\
TT vs. CC & 0.72 & $0.89(0.49-1.64)$ & $44 \%$ & 0.18 \\
CC vs. CT + TT & 0.53 & $1.07(0.87-1.31)$ & $33 \%$ & 0.22 \\
CT vs. CC + TT & 0.60 & $0.94(0.76-1.17)$ & $0 \%$ & 0.43 \\
TT vs. CC + CT & 0.74 & $0.90(0.49-1.65)$ & $41 \%$ & 0.19 \\
C vs. T & 0.45 & $1.17(0.77-1.77)$ & $59 \%$ & 0.12
\end{tabular}


Table II. Continued.

F, Hepatocellular cancer $\left(n=2^{a}\right)$

\begin{tabular}{|c|c|c|c|c|}
\hline Variable & P-value & OR $(95 \% \mathrm{Cl})$ & I-square (\%) & $\begin{array}{c}\text { P-value } \\
\text { for the } \\
\text { heterogeneity }\end{array}$ \\
\hline $\mathrm{CC}$ vs. $\mathrm{CT}$ & 0.37 & $0.23(0.01-5.69)$ & $92 \%$ & 0.0005 \\
\hline CT vs. TT & 0.62 & $0.88(0.54-1.44)$ & $0 \%$ & 0.79 \\
\hline TT vs. CC & 0.93 & $1.05(0.36-3.04)$ & $10 \%$ & 0.33 \\
\hline $\mathrm{CC}$ vs. CT + TT & 0.46 & $0.51(0.09-3.03)$ & $80 \%$ & 0.03 \\
\hline $\mathrm{CT}$ vs. $\mathrm{CC}+\mathrm{TT}$ & 0.16 & $0.55(0.23-1.27)$ & $84 \%$ & 0.01 \\
\hline TT vs. $\mathrm{CC}+\mathrm{CT}$ & 0.95 & $0.99(0.62-1.58)$ & $36 \%$ & 0.21 \\
\hline C vs. T & 0.28 & $0.48(0.13-1.80)$ & $96 \%$ & 0.00001 \\
\hline \multicolumn{5}{|c|}{ G, Lymphoma $\left(\mathrm{n}=2^{\mathrm{a}}\right)$} \\
\hline CC vs. CT & 0.42 & $0.82(0.50-1.34)$ & $0 \%$ & 0.59 \\
\hline CT vs. TT & 0.28 & $1.85(0.61-5.61)$ & $0 \%$ & 0.32 \\
\hline TT vs. CC & 0.34 & $0.60(0.21-1.72)$ & $24 \%$ & 0.25 \\
\hline $\mathrm{CC}$ vs. $\mathrm{CT}+\mathrm{TT}$ & 0.81 & $0.95(0.59-1.51)$ & $0 \%$ & 0.43 \\
\hline CT vs. $\mathrm{CC}+\mathrm{TT}$ & 0.45 & $1.21(0.74-1.98)$ & $0 \%$ & 0.88 \\
\hline TT vs. $\mathrm{CC}+\mathrm{CT}$ & 0.31 & $0.58(0.21-1.65)$ & $23 \%$ & 0.26 \\
\hline C vs. T & 0.88 & $0.95(0.51-1.76)$ & $54 \%$ & 0.14 \\
\hline \multicolumn{5}{|c|}{$H$, Prostate cancer $\left(n=2^{a}\right)$} \\
\hline CC vs. CT & 0.87 & $1.07(0.48-2.41)$ & $78 \%$ & 0.03 \\
\hline CT vs. TT & 0.004 & $1.48(1.14-1.92)$ & $0 \%$ & 0.43 \\
\hline TT vs. CC & 0.0009 & $0.48(0.31-0.74)$ & $0 \%$ & 0.43 \\
\hline $\mathrm{CC}$ vs. CT + TT & 0.52 & $1.31(0.57-3.01)$ & $82 \%$ & 0.02 \\
\hline CT vs. CC + TT & 0.02 & $1.33(1.05-1.69)$ & $0 \%$ & 0.66 \\
\hline TT vs. $\mathrm{CC}+\mathrm{CT}$ & 0.0004 & $0.64(0.50-0.82)$ & $0 \%$ & 0.90 \\
\hline $\mathrm{C}$ vs. T & 0.20 & $1.29(0.87-1.90)$ & $66 \%$ & 0.09 \\
\hline
\end{tabular}

I, Breast cancer $\left(n=2^{a}\right)$

\begin{tabular}{lllrr}
\hline CC vs. CT & $\mathbf{0 . 0 5}$ & $\mathbf{1 . 2 1}(\mathbf{1 . 0 0 - 1 . 4 6 )}$ & $21 \%$ & 0.26 \\
CT vs. TT & 0.18 & $1.46(0.84-2.54)$ & $0 \%$ & 0.61 \\
TT vs. CC & $\mathbf{0 . 0 4}$ & $\mathbf{0 . 5 6}(\mathbf{0 . 3 3 - 0 . 9 7 )}$ & $7 \%$ & 0.91 \\
CC vs. CT + TT & $\mathbf{0 . 0 2}$ & $\mathbf{1 . 2 5}(\mathbf{1 . 0 4 - 1 . 5 1 )}$ & $21 \%$ & 0.30 \\
CT vs. CC + TT & 0.07 & $0.84(0.70-1.02)$ & $0 \%$ & 0.82 \\
TT vs. CC + CT & 0.06 & $0.60(0.35-1.02)$ & $0 \%$ & 0.41 \\
C vs. T & $\mathbf{0 . 0 0 7}$ & $\mathbf{1 . 2 5}(\mathbf{1 . 0 6 - 1 . 4 7 )}$ & & 0.26 \\
\hline
\end{tabular}

$\mathrm{J}$, Bladder cancer $\left(\mathrm{n}=1^{\mathrm{a}}\right)$

\begin{tabular}{|c|c|c|c|c|}
\hline CC vs. CT & 0.32 & $1.26(0.80-1.99)$ & NA & NA \\
\hline CT vs. TT & 0.38 & $0.92(0.76-1.11)$ & NA & NA \\
\hline TT vs. CC & 0.51 & $0.86(0.56-1.34)$ & NA & NA \\
\hline $\mathrm{CC}$ vs. $\mathrm{CT}+\mathrm{TT}$ & 0.43 & $1.19(0.77-1.85)$ & NA & NA \\
\hline $\mathrm{CT}$ vs. $\mathrm{CC}+\mathrm{TT}$ & 0.33 & $0.91(0.75-1.10)$ & NA & NA \\
\hline TT vs. $\mathrm{CC}+\mathrm{CT}$ & 0.54 & $1.06(0.88-1.28)$ & NA & NA \\
\hline C vs. T & 0.81 & $0.98(0.84-1.15)$ & NA & NA \\
\hline
\end{tabular}


Table II. Continued.

$\mathrm{K}$, Brain tumor $\left(\mathrm{n}=1^{\mathrm{a}}\right)$

\begin{tabular}{|c|c|c|c|c|}
\hline Variable & P-value & OR $(95 \% \mathrm{Cl})$ & I-square $(\%)$ & $\begin{array}{c}\text { P-value } \\
\text { for the } \\
\text { heterogeneity }\end{array}$ \\
\hline CC vs. CT & 0.06 & $1.35(0.99-1.83)$ & NA & NA \\
\hline CT vs. TT & 0.35 & $0.66(0.28-1.58)$ & NA & NA \\
\hline TT vs. CC & 0.20 & $1.76(0.75-4.14)$ & NA & NA \\
\hline $\mathrm{CC}$ vs. $\mathrm{CT}+\mathrm{TT}$ & 0.08 & $1.30(0.97-1.74)$ & NA & NA \\
\hline $\mathrm{CT}$ vs. $\mathrm{CC}+\mathrm{TT}$ & 0.05 & $0.74(0.55-1.00)$ & NA & NA \\
\hline TT vs. $\mathrm{CC}+\mathrm{CT}$ & 0.64 & $1.23(0.53-2.86)$ & NA & NA \\
\hline $\mathrm{C}$ vs. T & 0.17 & $1.20(0.93-1.55)$ & NA & NA \\
\hline \multicolumn{5}{|c|}{$\mathrm{L}$, Testicular tumor $\left(\mathrm{n}=1^{\mathrm{a}}\right)$} \\
\hline CC vs. CT & 0.31 & $0.87(0.66-1.14)$ & NA & NA \\
\hline CT vs. TT & 0.66 & $1.21(0.51-2.85)$ & NA & NA \\
\hline TT vs. CC & 0.91 & $0.95(0.41-2.20)$ & NA & NA \\
\hline $\mathrm{CC}$ vs. $\mathrm{CT}+\mathrm{TT}$ & 0.35 & $0.88(0.67-1.15)$ & NA & NA \\
\hline $\mathrm{CT}$ vs. $\mathrm{CC}+\mathrm{TT}$ & 0.30 & $1.15(0.88-1.52)$ & NA & NA \\
\hline TT vs. $\mathrm{CC}+\mathrm{CT}$ & 0.84 & $0.92(0.40-2.12)$ & NA & NA \\
\hline C vs. T & 0.43 & $0.91(0.72-1.15)$ & NA & NA \\
\hline \multicolumn{5}{|c|}{ M, Leukemia $\left(n=1^{a}\right)$} \\
\hline CC vs. CT & 0.005 & $5.35(1.64-17.47)$ & NA & NA \\
\hline CT vs. TT & NA & NA & NA & NA \\
\hline TT vs. CC & NA & NA & NA & NA \\
\hline $\mathrm{CC}$ vs. $\mathrm{CT}+\mathrm{TT}$ & 0.01 & $4.67(1.42-15.31)$ & NA & NA \\
\hline $\mathrm{CT}$ vs. $\mathrm{CC}+\mathrm{TT}$ & 0.005 & $0.19(0.06-0.61)$ & NA & NA \\
\hline TT vs. $\mathrm{CC}+\mathrm{CT}$ & NA & $1.11(0.86-1.44)$ & NA & NA \\
\hline C vs. T & 0.06 & $1.97(0.96-4.02)$ & NA & NA \\
\hline
\end{tabular}

$\mathrm{N}$, Renal cell carcinoma

$\left(\mathrm{n}=1^{\mathrm{a}}\right)$

\begin{tabular}{|c|c|c|c|c|}
\hline CC vs. CT & 0.11 & $0.63(0.36-1.11)$ & NA & NA \\
\hline CT vs. TT & 0.55 & $0.93(0.73-1.18)$ & NA & NA \\
\hline TT vs. CC & 0.06 & $1.71(0.99-2.95)$ & NA & NA \\
\hline $\mathrm{CC}$ vs. $\mathrm{CT}+\mathrm{TT}$ & 0.06 & $0.60(0.35-1.03)$ & NA & NA \\
\hline $\mathrm{CT}$ vs. $\mathrm{CC}+\mathrm{TT}$ & 0.76 & $0.96(0.76-1.22)$ & NA & NA \\
\hline TT vs. $\mathrm{CC}+\mathrm{CT}$ & 0.26 & $1.14(0.91-1.44)$ & NA & NA \\
\hline C vs. T & 0.10 & $0.85(0.70-1.03)$ & NA & NA \\
\hline
\end{tabular}

O, Caucasian $\left(n=15^{a}\right)$

\begin{tabular}{lcccc}
\hline CC vs. CT & 0.85 & $0.98(0.75-1.27)$ & $81 \%$ & 0.00001 \\
CT vs. TT & $\mathbf{0 . 0 3}$ & $\mathbf{0 . 8 2}(\mathbf{0 . 6 8 - 0 . 9 8 )}$ & $46 \%$ & 0.03 \\
TT vs. CC & 0.84 & $1.03(0.81-1.30)$ & $0 \%$ & 0.50 \\
CC vs. CT + TT & 0.56 & $1.07(0.85-1.34)$ & $67 \%$ & 0.00001 \\
CT vs. CC + TT & $\mathbf{0 . 0 2}$ & $\mathbf{0 . 7 9}(\mathbf{0 . 6 6 - 0 . 9 6 )}$ & $4 \%$ & 0.0003 \\
TT vs. CC + CT & 0.10 & $0.83(0.67-1.04)$ & $90 \%$ & 0.41 \\
C vs. T & 0.84 & $1.03(0.80-1.33)$ & 90001
\end{tabular}


Table II. Continued.

$\mathrm{P}, \operatorname{Asian}\left(\mathrm{n}=12^{\mathrm{a}}\right)$

P-value

for the

\begin{tabular}{|c|c|c|c|c|}
\hline Variable & P-value & OR $(95 \% \mathrm{Cl})$ & I-square $(\%)$ & heterogeneity \\
\hline CC vs. CT & 0.26 & $1.22(0.87-1.72)$ & $71 \%$ & 0.00001 \\
\hline CT vs. TT & 0.11 & $0.90(0.79-1.02)$ & $47 \%$ & 0.04 \\
\hline TT vs. CC & 0.38 & $0.83(0.54-1.27)$ & $80 \%$ & 0.00001 \\
\hline $\mathrm{CC}$ vs. $\mathrm{CT}+\mathrm{TT}$ & 0.34 & $1.19(0.83-1.72)$ & $77 \%$ & 0.00001 \\
\hline CT vs. CC + TT & 0.006 & $0.89(0.82-0.97)$ & $36 \%$ & 0.11 \\
\hline TT vs. $\mathrm{CC}+\mathrm{CT}$ & 0.62 & $1.04(0.89-1.21)$ & $66 \%$ & 0.0007 \\
\hline C vs. T & 0.90 & $1.01(0.87-1.18)$ & $80 \%$ & 0.00001 \\
\hline
\end{tabular}

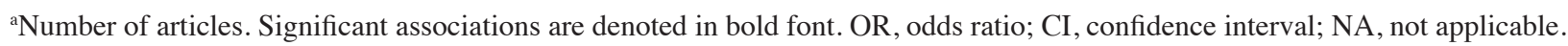

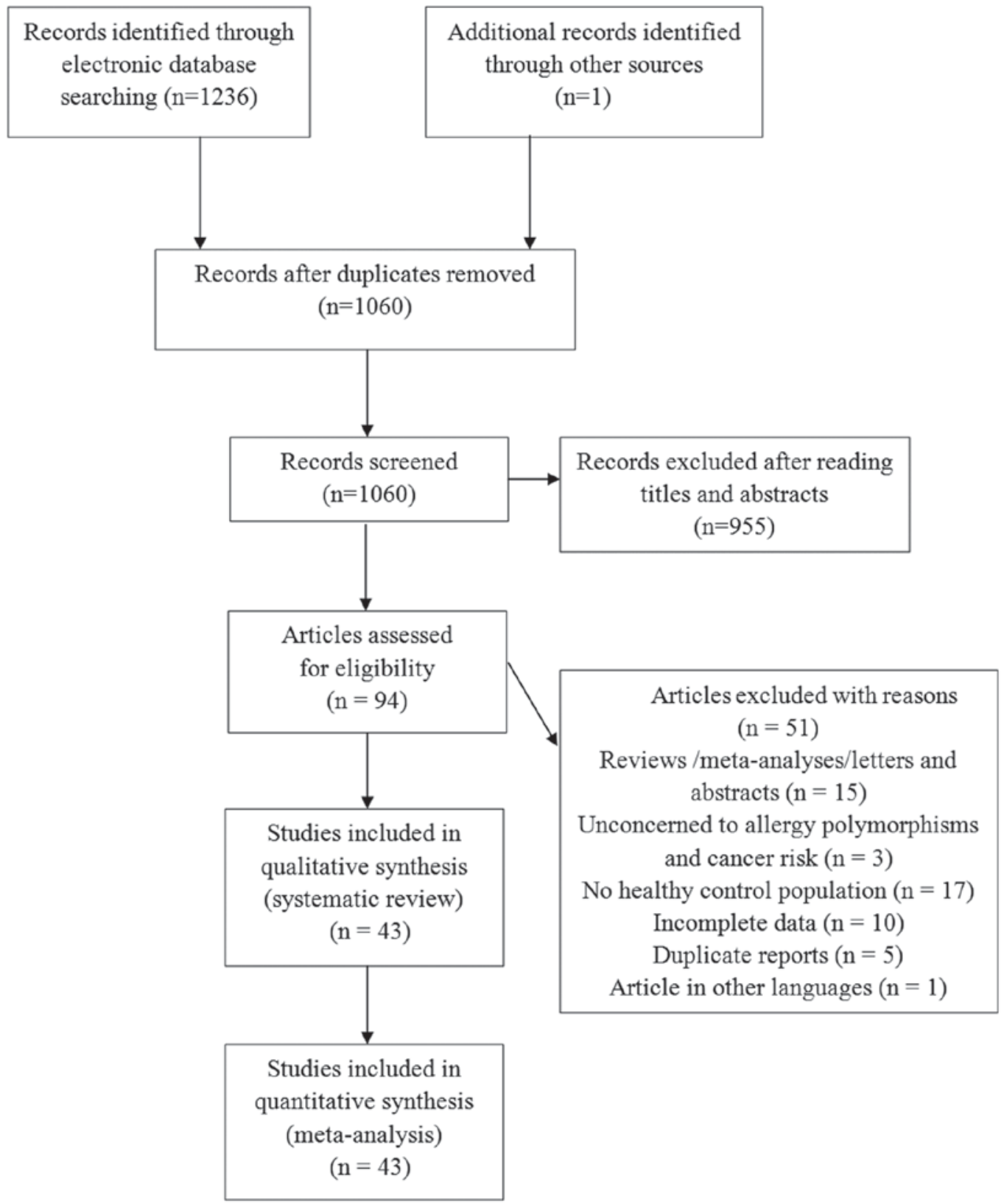

Figure 1. Flow diagram of included/excluded studies for the meta-analysis. 
A

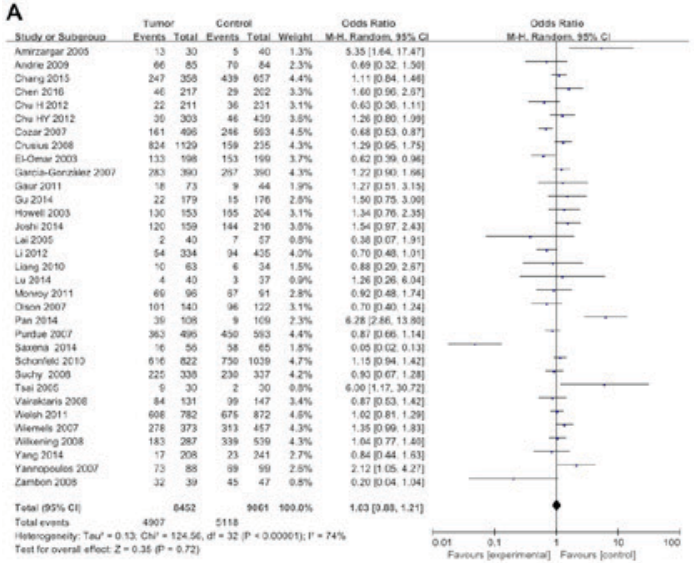

C

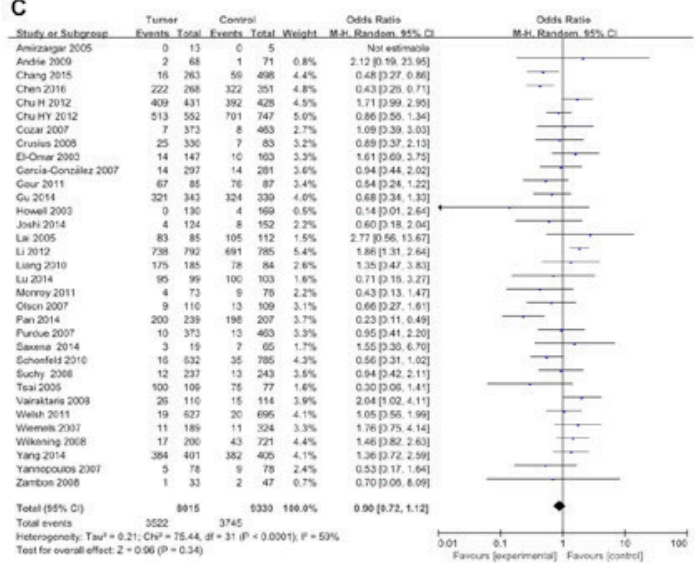

E

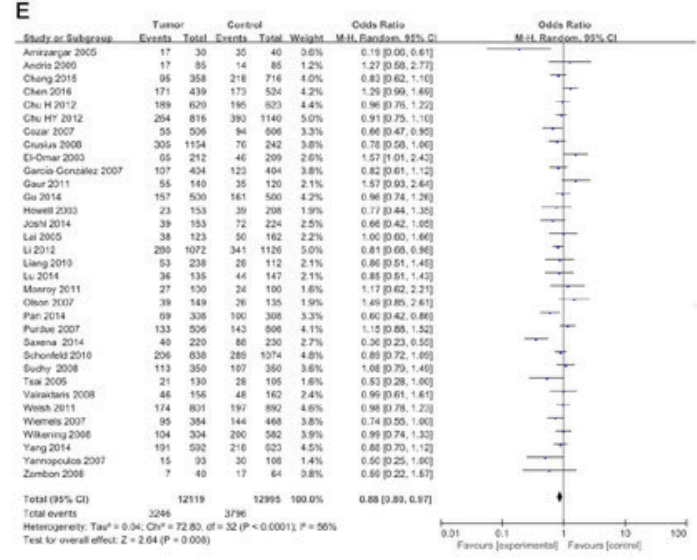

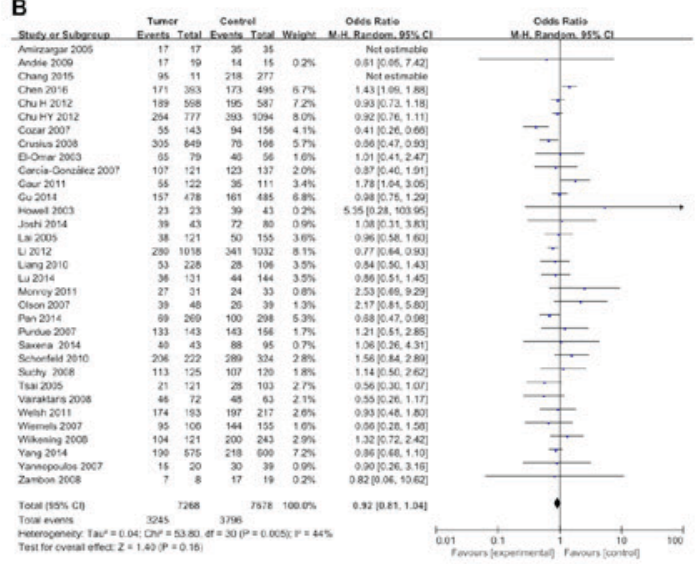

D

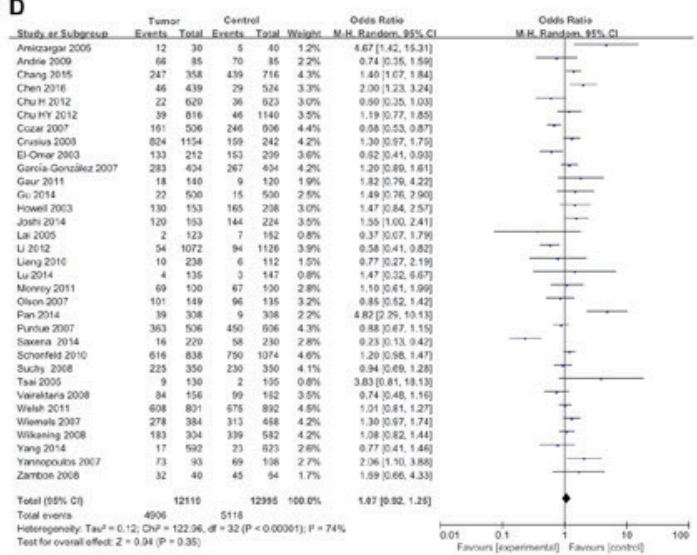

$\mathbf{F}$

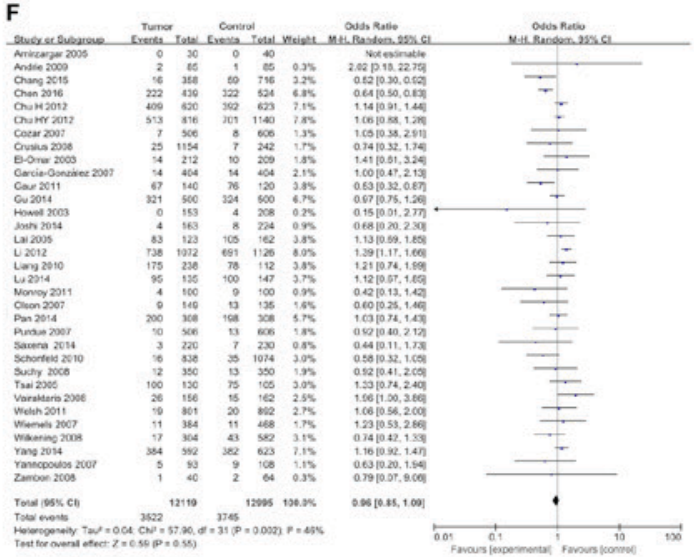

G

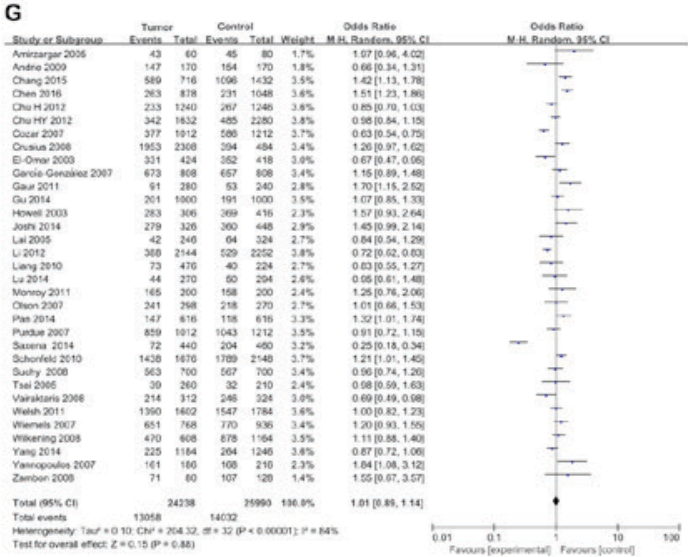

Figure 2. Forest plots of association between IL-4 rs2243250 polymorphism and cancer risk for all genetic models. (A) CC vs. CT. (B) CT vs. TT. (C) TT vs. CC. (D) CC vs. CT. (E) CT vs. CC/TT. (F) TT vs. CC/CT. (G) C vs. T. The squares and horizontal lines correspond to the study-specific OR and 95\% CI. The area of the squares reflects the weight (inverse of the variance). The diamond represents the summary OR and 95\% CI. IL, interleukin; OR, odds ratio; CI, confidence interval. 


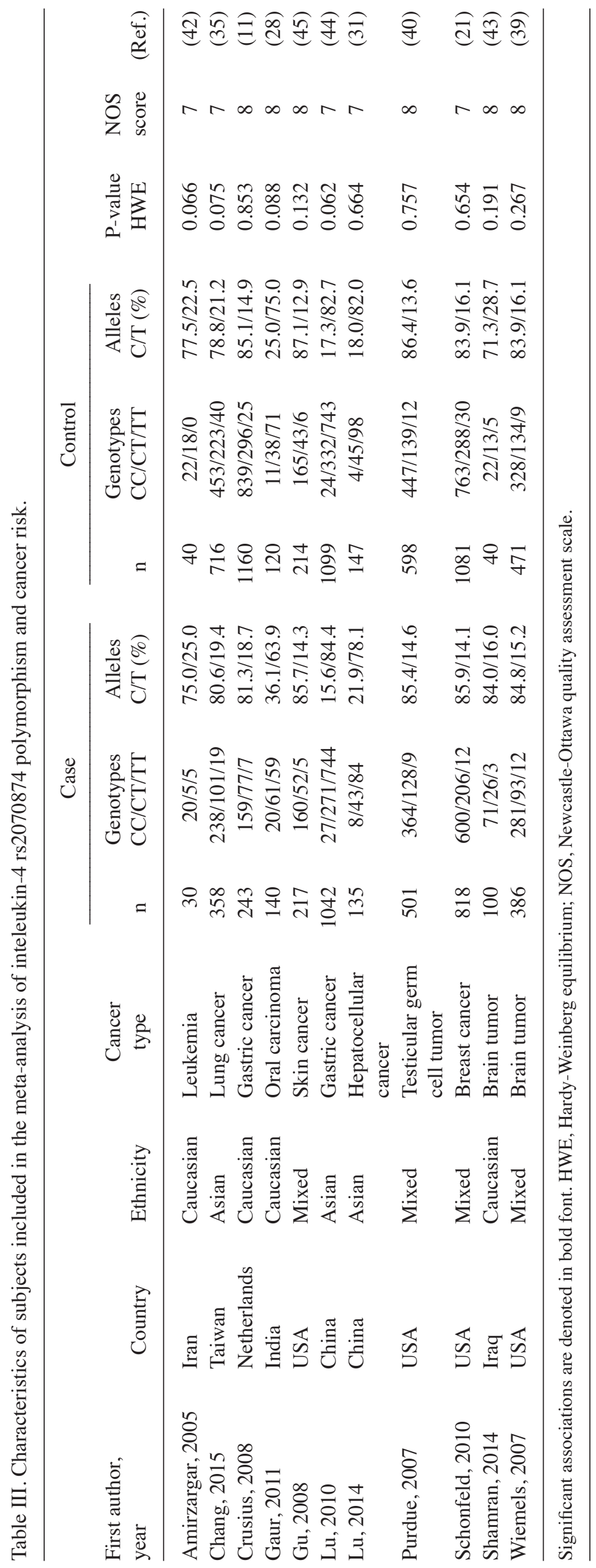


Table IV. Subgroup analyses for interleukin-4 rs2070874polymorphism and cancer risk.

A, Gastric cancer $\left(n=3^{a}\right)$

\begin{tabular}{|c|c|c|c|c|}
\hline Variable & P-value & OR $(95 \% \mathrm{Cl})$ & I-square (\%) & $\begin{array}{c}\text { P-value } \\
\text { for the } \\
\text { heterogeneity }\end{array}$ \\
\hline CC vs. CT & 0.91 & $1.03(0.60-1.76)$ & $59 \%$ & 0.09 \\
\hline CT vs. TT & 0.07 & $0.85(0.71-1.01)$ & $0 \%$ & 0.52 \\
\hline TT vs. CC & 0.67 & $0.91(0.59-1.41)$ & $25 \%$ & 0.26 \\
\hline CC vs. CT + TT & 0.94 & $1.02(0.60-1.74)$ & $60 \%$ & 0.08 \\
\hline CT vs. CC + TT & 0.85 & $1.04(0.72-1.49)$ & $75 \%$ & 0.85 \\
\hline TT vs. CC + CT & 0.11 & $1.15(0.97-1.36)$ & $5 \%$ & 0.35 \\
\hline C vs. T & 0.35 & $0.90(0.72-1.12)$ & $53 \%$ & 0.12 \\
\hline \multicolumn{5}{|c|}{$\mathrm{B}$, Brain tumor $\left(\mathrm{n}=2^{\mathrm{a}}\right)$} \\
\hline CC vs. CT & 0.10 & $1.27(0.95-1.70)$ & $0 \%$ & 0.55 \\
\hline CT vs. TT & 0.86 & $1.17(0.19-7.13)$ & $75 \%$ & 0.05 \\
\hline TT vs. CC & 0.62 & $0.59(0.07-4.70)$ & $82 \%$ & 0.02 \\
\hline $\mathrm{CC}$ vs. $\mathrm{CT}+\mathrm{TT}$ & 0.11 & $1.25(0.95-1.65)$ & $41 \%$ & 0.19 \\
\hline CT vs. CC + TT & 0.30 & $0.52(0.15-1.77)$ & $78 \%$ & 0.03 \\
\hline TT vs. $\mathrm{CC}+\mathrm{CT}$ & 0.67 & $0.65(0.09-4.74)$ & $81 \%$ & 0.02 \\
\hline C vs. T & 0.29 & $1.42(0.74-2.73)$ & $75 \%$ & 0.05 \\
\hline \multicolumn{5}{|c|}{ C, Leukemia $\left(n=1^{a}\right)$} \\
\hline CC vs. CT & 0.05 & 3.27 (1.02-10.45) & NA & NA \\
\hline CT vs. TT & 0.02 & $0.03(0.00-0.57)$ & NA & NA \\
\hline TT vs. CC & 0.10 & $12.07(0.63-232.12)$ & NA & NA \\
\hline CC vs. CT + TT & 0.33 & $1.64(0.61-4.37)$ & NA & NA \\
\hline CT vs. CC + TT & 0.02 & 0.24 (0.08-0.77) & NA & NA \\
\hline TT vs. CC + CT & 0.06 & $17.47(0.93-329.53)$ & NA & NA \\
\hline C vs. T & 0.73 & $0.87(0.40-1.91)$ & NA & NA \\
\hline
\end{tabular}

$\mathrm{D}$, Lung cancer $\left(\mathrm{n}=1^{\mathrm{a}}\right)$

\begin{tabular}{|c|c|c|c|c|}
\hline CC vs. CT & 0.30 & $1.16(0.87-1.54)$ & NA & NA \\
\hline CT vs. TT & 0.88 & $0.95(0.53-1.73)$ & NA & NA \\
\hline TT vs. CC & 0.73 & $0.90(0.51-1.60)$ & NA & NA \\
\hline $\mathrm{CC}$ vs. $\mathrm{CT}+\mathrm{TT}$ & 0.30 & $1.15(0.88-1.50)$ & NA & NA \\
\hline $\mathrm{CT}$ vs. $\mathrm{CC}+\mathrm{TT}$ & 0.32 & $0.87(0.66-1.15)$ & NA & NA \\
\hline TT vs. $\mathrm{CC}+\mathrm{CT}$ & 0.85 & $0.95(0.54-1.66)$ & NA & NA \\
\hline C vs. T & 0.35 & $1.11(0.89-1.39)$ & NA & NA \\
\hline \multicolumn{5}{|c|}{$\mathrm{E}$, Oral carcinoma $\left(\mathrm{n}=1^{\mathrm{a}}\right)$} \\
\hline $\mathrm{CC}$ vs. $\mathrm{CT}$ & 0.77 & $1.13(0.49-2.62)$ & NA & NA \\
\hline CT vs. TT & 0.02 & $1.93(1.13-3.29)$ & NA & NA \\
\hline TT vs. CC & 0.06 & $0.46(0.20-1.03)$ & NA & NA \\
\hline $\mathrm{CC}$ vs. $\mathrm{CT}+\mathrm{TT}$ & 0.21 & $1.65(0.76-3.60)$ & NA & NA \\
\hline $\mathrm{CT}$ vs. $\mathrm{CC}+\mathrm{TT}$ & 0.05 & $1.67(1.00-2.77)$ & NA & NA \\
\hline TT vs. $\mathrm{CC}+\mathrm{CT}$ & 0.006 & $0.50(0.31-0.82)$ & NA & NA \\
\hline C vs. T & 0.007 & $1.69(1.16-2.48)$ & NA & NA \\
\hline
\end{tabular}


Table IV. Continued.

F, Breast cancer $\left(n=1^{a}\right)$

\begin{tabular}{|c|c|c|c|c|}
\hline Variable & P-value & OR $(95 \% \mathrm{Cl})$ & I-square (\%) & $\begin{array}{c}\text { P-value } \\
\text { for the } \\
\text { heterogeneity }\end{array}$ \\
\hline CC vs. CT & 0.37 & $1.10(0.89-1.35)$ & NA & NA \\
\hline CT vs. TT & 0.10 & $1.79(0.89-3.58)$ & NA & NA \\
\hline TT vs. CC & 0.05 & $0.51(0.26-1.00)$ & NA & NA \\
\hline $\mathrm{CC}$ vs. $\mathrm{CT}+\mathrm{TT}$ & 0.18 & $1.15(0.94-1.41)$ & NA & NA \\
\hline $\mathrm{CT}$ vs. $\mathrm{CC}+\mathrm{TT}$ & 0.47 & $0.93(0.75-1.14)$ & NA & NA \\
\hline TT vs. $\mathrm{CC}+\mathrm{CT}$ & 0.06 & $0.52(0.27-1.03)$ & NA & NA \\
\hline C vs. T & 0.08 & $1.17(0.98-1.40)$ & NA & NA \\
\hline \multicolumn{5}{|c|}{$\mathrm{G}$, Testicular tumor $\left(\mathrm{n}=1^{\mathrm{a}}\right)$} \\
\hline CC vs. CT & 0.38 & $0.88(0.67-1.17)$ & NA & NA \\
\hline CT vs. TT & 0.65 & $1.23(0.50-3.01)$ & NA & NA \\
\hline TT vs. CC & 0.85 & $0.92(0.38-2.21)$ & NA & NA \\
\hline $\mathrm{CC}$ vs. $\mathrm{CT}+\mathrm{TT}$ & 0.43 & $0.90(0.69-1.18)$ & NA & NA \\
\hline $\mathrm{CT}$ vs. $\mathrm{CC}+\mathrm{TT}$ & 0.38 & $1.13(0.86-1.49)$ & NA & NA \\
\hline TT vs. $\mathrm{CC}+\mathrm{CT}$ & 0.80 & $0.89(0.37-2.14)$ & NA & NA \\
\hline C vs. T & 0.53 & $0.93(0.73-1.18)$ & NA & NA \\
\hline
\end{tabular}

$\mathrm{H}$, Skin cancer $\left(\mathrm{n}=1^{\mathrm{a}}\right)$

\begin{tabular}{|c|c|c|c|c|}
\hline CC vs. CT & 0.35 & $0.80(0.51-1.27)$ & NA & NA \\
\hline CT vs. TT & 0.56 & $1.45(0.41-5.08)$ & NA & NA \\
\hline TT vs. CC & 0.81 & $0.86(0.26-2.87)$ & NA & NA \\
\hline $\mathrm{CC}$ vs. $\mathrm{CT}+\mathrm{TT}$ & 0.42 & $0.83(0.54-1.29)$ & NA & NA \\
\hline $\mathrm{CT}$ vs. $\mathrm{CC}+\mathrm{TT}$ & 0.33 & $1.25(0.79-1.98)$ & NA & NA \\
\hline TT vs. $\mathrm{CC}+\mathrm{CT}$ & 0.74 & $0.82(0.25-2.72)$ & NA & NA \\
\hline C vs. T & 0.54 & $0.88(0.60-1.31)$ & NA & NA \\
\hline
\end{tabular}

I, Caucasian $\left(\mathrm{n}=4^{\mathrm{a}}\right)$

\begin{tabular}{lllll}
\hline CC vs. CT & 0.48 & $1.25(0.67-2.33)$ & $66 \%$ & 0.03 \\
CT vs. TT & 0.78 & $1.16(0.41-3.29)$ & $70 \%$ & 0.02 \\
TT vs. CC & 0.67 & $0.78(0.24-2.51)$ & $72 \%$ & 0.01 \\
CC vs. CT + TT & 0.40 & $1.30(0.71-2.38)$ & $70 \%$ & 0.02 \\
CT vs. CC + TT & 0.92 & $0.97(0.55-1.73)$ & $73 \%$ & 0.01 \\
TT vs. CC + CT & 0.68 & $0.80(0.28-2.27)$ & $73 \%$ & 0.01 \\
C vs. T & 0.45 & $1.23(0.71-2.13)$ & $83 \%$ & 0.0006 \\
\hline
\end{tabular}

\section{$\mathrm{J}, \operatorname{Asian}\left(\mathrm{n}=3^{\mathrm{a}}\right)$}

\begin{tabular}{lllll}
\hline CC vs. CT & 0.12 & $1.22(0.95-1.56)$ & $0 \%$ & 0.77 \\
CT vs. TT & 0.07 & $0.86(0.72-1.01)$ & $0 \%$ & 0.49 \\
TT vs. CC & 0.35 & $0.83(0.57-1.22)$ & $0 \%$ & 0.54 \\
CC vs. CT + TT & 0.15 & $1.19(0.94-1.51)$ & $0 \%$ & 0.58 \\
CT vs. CC + TT & $\mathbf{0 . 0 3}$ & $\mathbf{0 . 8 5}(\mathbf{0 . 7 3 - 0 . 9 8 )}$ & $15 \%$ & 0.61 \\
TT vs. CC + CT & 0.17 & $1.12(0.95-1.32)$ & $54 \%$ & 0.11 \\
C vs. T & 0.81 & $1.03(0.83-1.26)$ & & 0.17 \\
\hline
\end{tabular}

${ }^{\text {an }}$ umber of articles. Significant associations are denoted in bold font. OR, odds ratio; CI, confidence interval; NA, not applicable. 


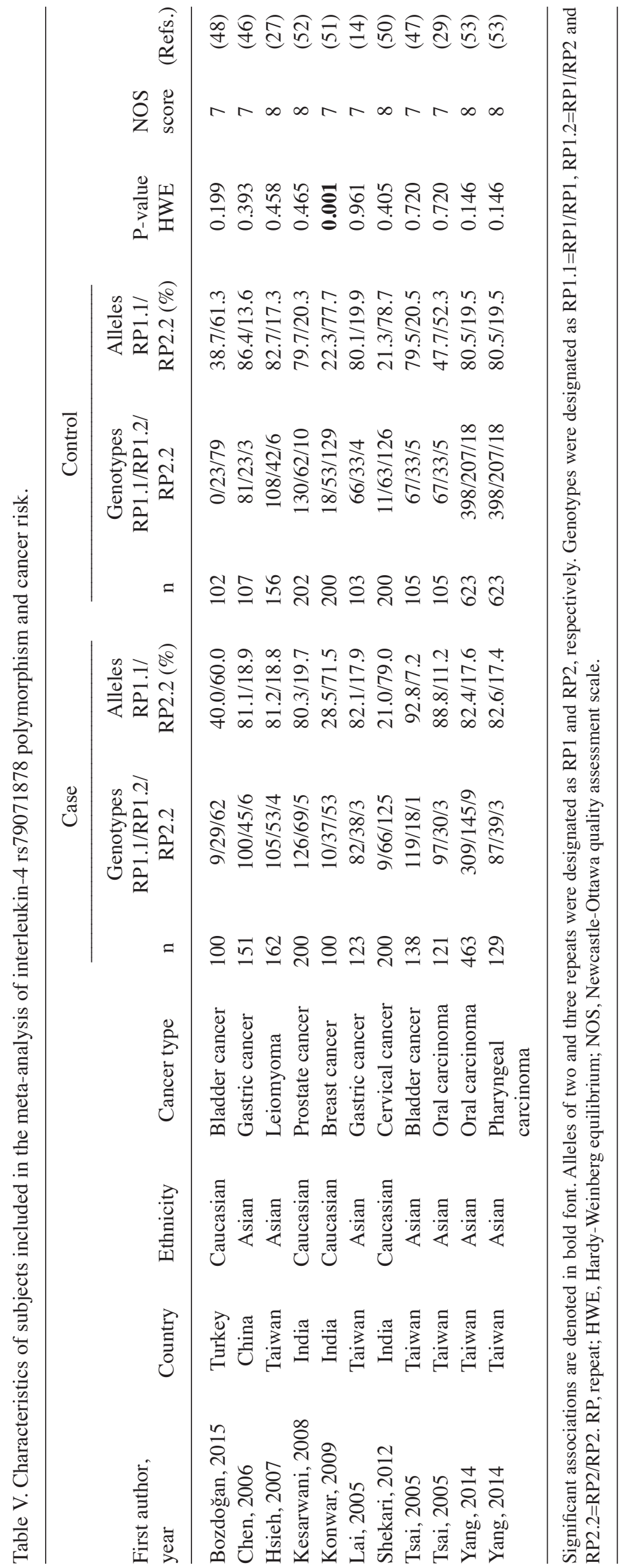



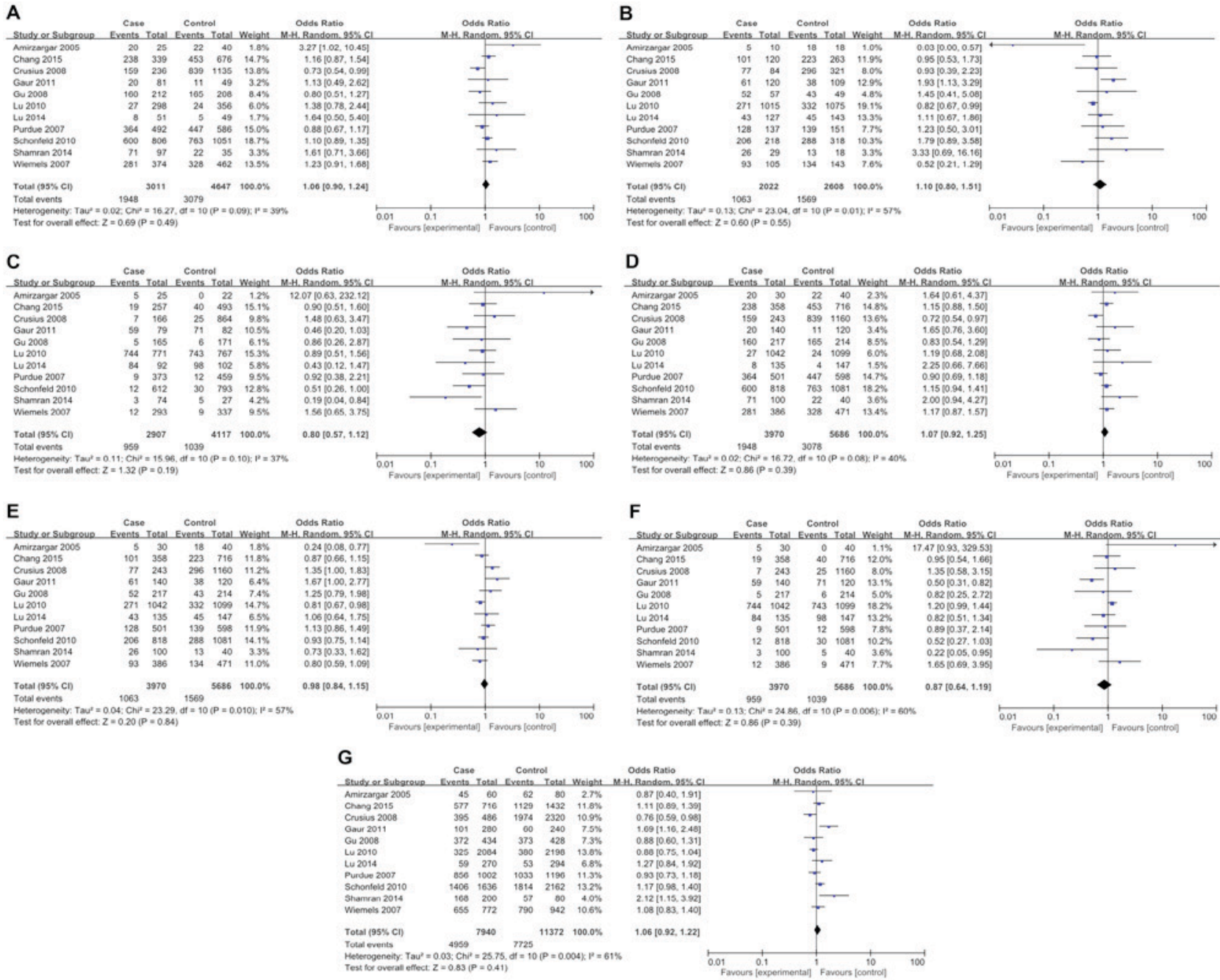

Figure 3. Forest plot of association between IL-4 rs2070874 polymorphism and cancer risk for all genetic models. (A) CC vs. CT. (B) CT vs. TT. (C) TT vs. CC. (D) CC vs. CT/TT. (E) CT vs. CC/TT. (F) TT vs. CC/CT. (G) C vs. T. The squares and horizontal lines correspond to the study specific OR and 95\% CI. The area of the squares reflects the weight (inverse of the variance). The diamond represents the summary OR and 95\% CI. IL, interleukin; OR, odds ratio; CI, confidence interval.

IL-4 rs2070874 polymorphism and the risk of cancer. For IL-4 rs2070874 polymorphism, 11 studies involving 3,970 patients and 5,686 controls were included. All relevant studies were in agreement with HWE (Table III). Inter-study heterogeneity was obvious in all comparisons and thus REMs were used for analyses. No significant association between IL-4 rs2070874 polymorphism and cancer risk was observed in all genetic models (Fig. 3). Further stratification analyses by cancer type revealed a significant association with leukemia (CC vs. CT: $\mathrm{P}=0.05$, $\mathrm{OR}=3.27,95 \%$ CI1.02-10.45; CT vs. TT: $\mathrm{P}=0.02, \mathrm{OR}=0.03,95 \%$ CI 0.00-0.57; and CT vs. CC/TT: $\mathrm{P}=0.02$, OR=0.24, 95\% CI 0.08-0.77), and oral carcinoma (CT vs. TT: $\mathrm{P}=0.02, \mathrm{OR}=1.93,95 \%$ CI1.13-3.29; CT vs. CC/TT: $\mathrm{P}=0.05, \mathrm{OR}=1.67,95 \%$ CI 1.00-2.77; TT vs. $\mathrm{CC} / \mathrm{CT}$ : $\mathrm{P}=0.006, \mathrm{OR}=0.50,95 \% \mathrm{CI} 0.31-0.82$; and $\mathrm{C}$ vs. $\mathrm{T}: \mathrm{P}=0.007, \mathrm{OR}=1.69,95 \%$ CI 1.16-2.48) (Table II). Nevertheless, no association was observed between rs2070874 polymorphism and other tumor types (Table III). In the subgroup analyses by ethnicity, a significant association was found in Asian populations (CT vs. CC/TT: $\mathrm{P}=0.03$, $\mathrm{OR}=0.85$, 95\% CI 0.73-0.98), but not in Caucasian populations (Table IV).
IL-4 rs79071878 polymorphism and the risk of cancer. A total of 10 studies with 1,896 patients and 2,526 controls were involved in the present analyses for IL-4 rs79071878 polymorphism and cancer risk. HWE test revealed that only one study deviated from HWE (Table V). IL-4 VNTR is a 70 bp repeat. Alleles of two and three repeats were designated as repeat 1 (RP1) and repeat 2 (RP2), respectively, and genotypes of RP1/RP1, RP1/RP2 and RP2/RP2 were designated as RP1.1, RP1.2 and RP2.2, respectively. For RP1.2 vs. RP2.2, RP2.2 vs. RP1.1 and RP2.2 vs. RP1.1/RP1.2, FEMs were selected for analyses since only mild inter-study heterogeneity was observed. In contrast, for RP1.1 vs. RP1.2, RP1.1 vs. RP1.2/RP2.2, RP1.2 vs. RP1.1/RP2.2 and RP1 vs. RP2, REMs were used because heterogeneity between studies was significant. The results demonstrated an apparent correlation between IL-4 rs79071878 polymorphism and cancer risk (RP1.2 vs. RP2.2: $\mathrm{P}=0.008, \mathrm{OR}=1.40,95 \%$ CI 1.09-1.79; RP2.2 vs. RP1.1: $\mathrm{P}=0.006, \mathrm{OR}=0.62,95 \% \mathrm{CI} 0.44-0.87, \mathrm{RP} 2.2$ vs. RP1.1/RP1.2: $\mathrm{P}=0.002, \mathrm{OR}=0.69,95 \% \mathrm{CI} 0.55-0.88$; and RP1.1 vs. RP2.2: $\mathrm{P}=0.05, \mathrm{OR}=1.26,95 \%$ CI 1.00-1.58; Fig. 4). Further analyses by cancer type subgroup revealed that the 
A

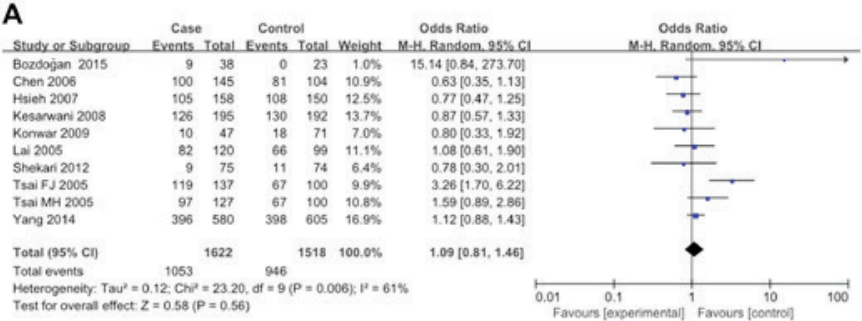

C

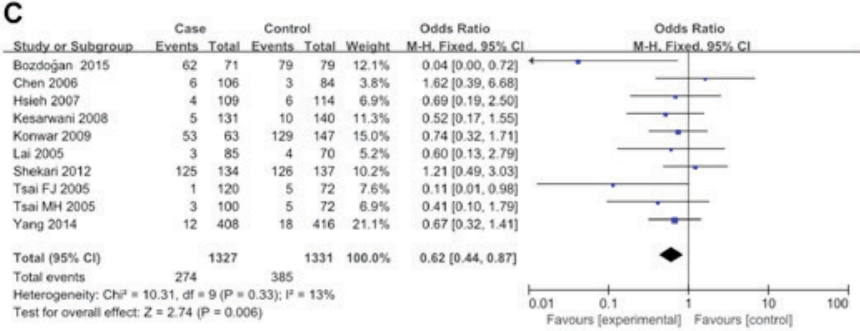

E

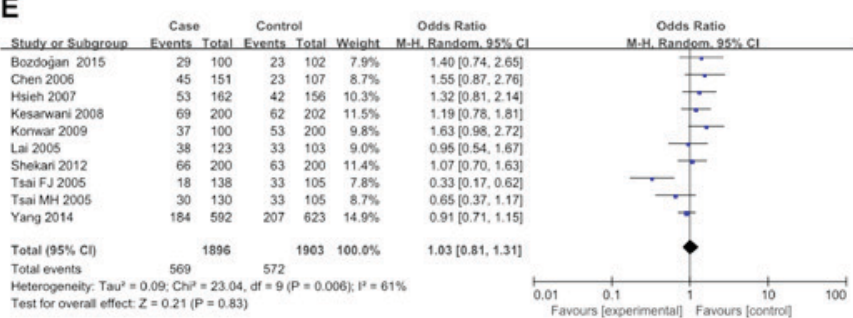

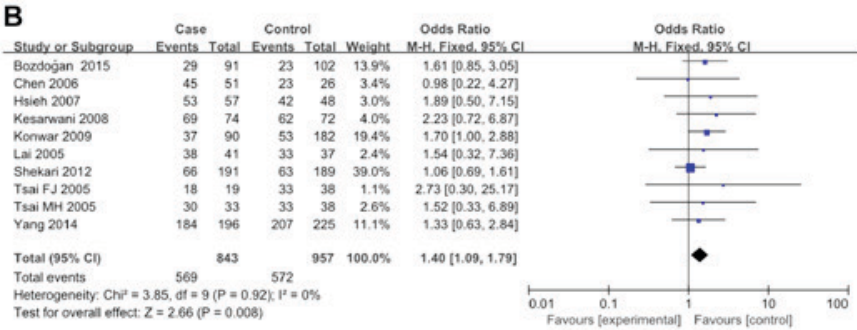

D

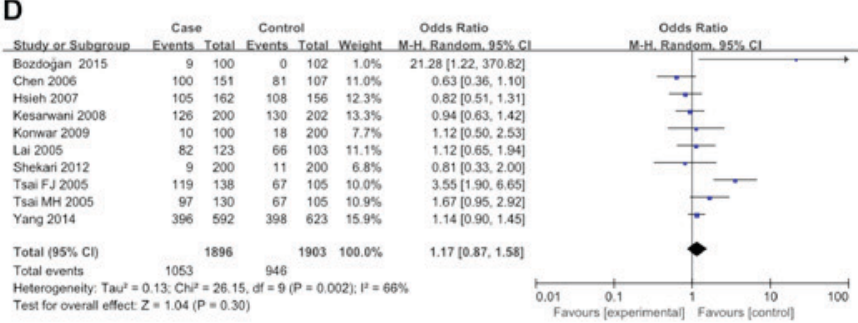

$\mathbf{F}$

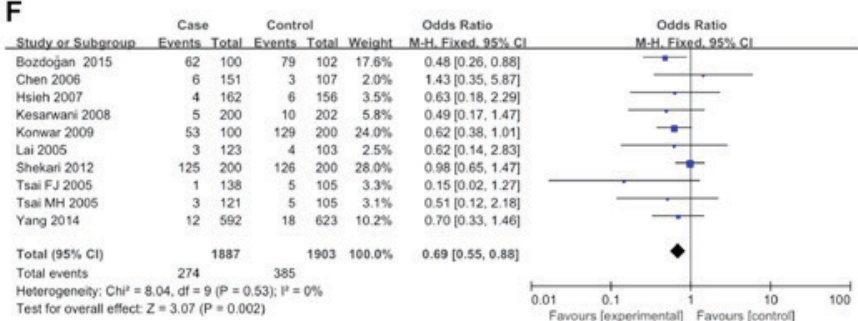

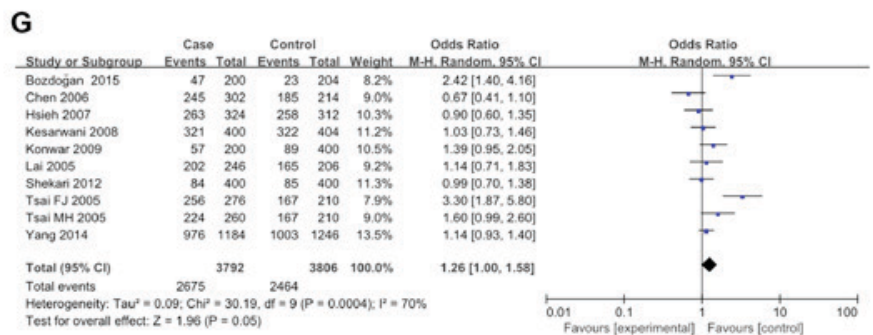

Figure 4. Forest plot of association between IL-4 rs79071878 polymorphism and cancer risk for all genetic models. (A) RP1.1 vs. RP1.2. (B) RP1.2 vs. RP2.2. (C) RP2.2 vs. RP1.1. (D) RP1.1 vs. RP1.2/RP2.2. (E) RP1.2 vs. RP1.1/RP2.2. (F) RP2.2 vs. RP1.1/RP1.2. (G) RP1 vs. RP2. The squares and horizontal lines correspond to the study specific OR and $95 \% \mathrm{CI}$. The area of the squares reflects the weight (inverse of the variance). The diamond represents the summary OR and $95 \% \mathrm{CI}$. Alleles of two and three repeats were designated as RP1 and RP2, respectively. Genotypes were designated as RP1.1=RP1/RP1, RP1.2=RP1/RP2 and RP2.2=RP2/RP2. IL, interleukin; RP, repeat; OR, odds ratio; $\mathrm{CI}$, confidence interval.

rs79071878 polymorphism was associated with an increased risk of bladder cancer (RP1.1 vs. RP1.2: $\mathrm{P}<0.0001, \mathrm{OR}=3.78$, 95\% CI 2.03-7.05; RP2.2 vs. RP1.1: $\mathrm{P}=0.002, \mathrm{OR}=0.07,95 \%$ CI 0.01-0.38; RP1.1 vs. RP1.2/RP2.2: $\mathrm{P}<0.0001, \mathrm{OR}=4.28$, 95\% CI 2.35-7.81; and RP2.2 vs. RP1.1/RP1.2: $\mathrm{P}=0.004$, $\mathrm{OR}=0.42,95 \% \mathrm{CI}$ 0.24-0.76) and breast cancer (RP1.2 vs. RP2.2: $\mathrm{P}=0.05, \mathrm{OR}=1.70,95 \%$ CI 1.00-2.88) (Table VI). However, no significant association was observed in other types of cancer. Furthermore, stratified analysis by ethnicity yielded a significant association for the IL-4 rs79071878 polymorphism with cancer risk in the Asian ethnicity (RP1.2 vs. $\mathrm{RP} 2.2$ : $\mathrm{P}=0.03, \mathrm{OR}=1.38,95 \%$ CI 1.04-1.83; and RP2.2 vs. RP1.1/RP1.2: $\mathrm{P}=0.01, \mathrm{OR}=0.71,95 \%$ CI 0.54-0.93). However, no evidence for any associations between IL-4 rs79071878 polymorphism and cancer risk was detected in the Caucasian ethnicity (Table VI).

Sensitivity analysis and publication bias. Sensitivity analyses were performed by removing one individual study from the analysis at a time. For IL-4 rs2243250 polymorphism, when the study of Chen et al (22) was omitted, the comparison in $\mathrm{CT}$ vs. TT yielded positive result $(\mathrm{P}=0.03, \mathrm{OR}=0.88,95 \%$ CI 0.79-0.98). For IL-4 rs2070874 and rs79071878 polymorphisms, however, removing individual studies did not impact the overall results. Publication bias was evaluated with funnel plots, and visual inspection of the funnel plots for all investigated polymorphisms indicated that there was no significant publication bias in the present meta-analysis.

\section{Discussion}

Cancer is a major public health problem with extremely high morbidity and mortality. Certain cytokine gene polymorphisms may serve crucial roles in cancer pathogenesis. Among these, IL-4 rs2243250, rs2070874 and rs79071878 polymorphisms are three intensively studied variants. Previous studies have demonstrated that the T allele of IL-4 rs2243250 and rs2070874 polymorphisms can increase binding of nuclear transcription factors to the promoter region of the IL-4 gene, and thus lead to increased transcription of 
Table VI. Subgroup analyses for inteleukin-4 rs79071878polymorphism and cancer risk.

A, Bladder cancer $\left(n=2^{a}\right)$

\begin{tabular}{|c|c|c|c|c|}
\hline Variable & P-value & OR $(95 \% \mathrm{Cl})$ & I-square $(\%)$ & $\begin{array}{c}\text { P-value } \\
\text { for the } \\
\text { heterogeneity }\end{array}$ \\
\hline RP1.1 vs. RP1.2 & 0.56 & $1.09(0.81-1.46)$ & $8 \%$ & 0.30 \\
\hline RP1.2 vs. RP2.2 & 0.008 & $1.40(1.09-1.79)$ & $0 \%$ & 0.65 \\
\hline RP2.2 vs. RP1.1 & 0.006 & $0.62(0.44-0.87)$ & $0 \%$ & 0.57 \\
\hline RP1.1 vs. RP1.2/ RP2.2 & 0.30 & $1.17(0.87-1.58)$ & $35 \%$ & 0.21 \\
\hline RP1.2 vs. RP1.1/RP2.2 & 0.83 & $1.03(0.81-1.13)$ & $90 \%$ & 0.002 \\
\hline RP2.2 vs. RP1.1/ RP1.2 & 0.002 & $0.69(0.55-0.88)$ & $6 \%$ & 0.30 \\
\hline RP1 vs. RP2 & 0.005 & $1.26(1.00-1.58)$ & $0 \%$ & 0.44 \\
\hline \multicolumn{5}{|l|}{$B$, Gastric cancer $\left(n=2^{a}\right)$} \\
\hline RP1.1 vs. RP1.2 & 0.36 & $0.83(0.49-1.40)$ & $40 \%$ & 0.20 \\
\hline RP1.2 vs. RP2.2 & 0.73 & $1.21(0.42-3.50)$ & $0 \%$ & 0.68 \\
\hline RP2.2 vs. RP1.1 & 0.94 & $1.04(0.38-2.85)$ & $0 \%$ & 0.35 \\
\hline RP1.1 vs. RP1.2/RP2.2 & 0.55 & $0.84(0.48-1.48)$ & $52 \%$ & 0.15 \\
\hline RP1.2 vs. RP1.1/RP2.2 & 0.35 & $1.21(0.81-1.81)$ & $30 \%$ & 0.23 \\
\hline RP2.2 vs. RP1.1/RP1.2 & 0.97 & $0.98(0.36-2.69)$ & $0 \%$ & 0.43 \\
\hline RP1 vs. RP2 & 0.63 & $0.88(0.52-1.47)$ & $57 \%$ & 0.13 \\
\hline
\end{tabular}

C, Leiomyoma $\left(n=1^{a}\right)$

\begin{tabular}{|c|c|c|c|c|}
\hline RP1.1 vs. RP1.2 & 0.29 & $0.77(0.47-1.25)$ & NA & NA \\
\hline RP1.2 vs. RP2.2 & 0.35 & $1.89(0.50-7.15)$ & NA & NA \\
\hline RP2.2 vs. RP1.1 & 0.57 & $0.69(0.19-2.50)$ & NA & NA \\
\hline RP1.1 vs. RP1.2/RP2.2 & 0.40 & $0.82(0.51-1.31)$ & NA & NA \\
\hline RP1.2 vs. RP1.1/RP2.2 & 0.26 & $1.32(0.81-2.14)$ & NA & NA \\
\hline RP2.2 vs. RP1.1/RP1.2 & 0.49 & $0.63(0.18-2.29)$ & NA & NA \\
\hline RP1 vs. RP2 & 0.62 & $0.90(0.60-1.35)$ & NA & NA \\
\hline
\end{tabular}

$\mathrm{D}$, Oral carcinoma $\left(\mathrm{n}=1^{\mathrm{a}}\right)$

\begin{tabular}{|c|c|c|c|c|}
\hline RP1.1 vs. RP1.2 & 0.12 & $1.59(0.89-2.86)$ & NA & NA \\
\hline RP1.2 vs. RP2.2 & 0.59 & $1.52(0.33-6.89)$ & NA & NA \\
\hline RP2.2 vs. RP1.1 & 0.24 & $0.41(0.10-1.79)$ & NA & NA \\
\hline RP1.1 vs. RP1.2/RP2.2 & 0.07 & $1.67(0.95-2.92)$ & NA & NA \\
\hline RP1.2 vs. RP1.1/RP2.2 & 0.15 & $0.65(0.37-1.17)$ & NA & NA \\
\hline RP2.2 vs. RP1.1/RP1.2 & 0.36 & $0.51(0.12-2.18)$ & NA & NA \\
\hline RP1 vs. RP2 & 0.06 & $1.60(0.99-2.60)$ & NA & NA \\
\hline \multicolumn{5}{|l|}{$\mathrm{E}$, Prostate cancer $\left(\mathrm{n}=1^{\mathrm{a}}\right)$} \\
\hline RP1.1 vs. RP1.2 & 0.52 & $0.87(0.57-1.33)$ & NA & NA \\
\hline RP1.2 vs. RP2.2 & 0.16 & $2.23(0.72-6.87)$ & NA & NA \\
\hline RP2.2 vs. RP1.1 & 0.24 & $0.52(0.17-1.55)$ & NA & NA \\
\hline RP1.1 vs. RP1.2/RP2.2 & 0.78 & $0.94(0.63-1.42)$ & NA & NA \\
\hline RP1.2 vs. RP1.1/RP2.2 & 0.42 & $1.19(0.78-1.81)$ & NA & NA \\
\hline RP2.2 vs. RP1.1/RP1.2 & 0.20 & $0.49(0.17-1.47)$ & NA & NA \\
\hline RP1 vs. RP2 & 0.85 & $1.03(0.73-1.46)$ & NA & NA \\
\hline
\end{tabular}


Table VI. Continued.

F, Cervical cancer $\left(n=1^{a}\right)$

\begin{tabular}{|c|c|c|c|c|}
\hline Variable & P-value & OR $(95 \% \mathrm{Cl})$ & I-square $(\%)$ & $\begin{array}{c}\text { P-value } \\
\text { for the } \\
\text { heterogeneity }\end{array}$ \\
\hline RP1.1 vs. RP1.2 & 0.61 & $0.78(0.30-2.01)$ & NA & NA \\
\hline RP1.2 vs. RP2.2 & 0.80 & $1.06(0.69-1.61)$ & NA & NA \\
\hline RP2.2 vs. RP1.1 & 0.68 & $1.21(0.49-3.03)$ & NA & NA \\
\hline RP1.1 vs. RP1.2/RP2.2 & 0.65 & $0.81(0.33-2.00)$ & NA & NA \\
\hline RP1.2 vs. RP1.1/RP2.2 & 0.75 & $1.07(0.70-1.63)$ & NA & NA \\
\hline RP2.2 vs. RP1.1/RP1.2 & 0.92 & $0.98(0.65-1.47)$ & NA & NA \\
\hline RP1 vs. RP2 & 0.93 & $0.99(0.70-1.38)$ & NA & NA \\
\hline
\end{tabular}

$\mathrm{G}$, Breast cancer $\left(\mathrm{n}=1^{\mathrm{a}}\right)$

\begin{tabular}{lllll}
\hline RP1.1 vs. RP1.2 & 0.61 & $0.80(0.33-1.92)$ & NA & NA \\
RP1.2 vs. RP2.2 & $\mathbf{0 . 0 5}$ & $\mathbf{1 . 7 0 ( 1 . 0 0 - 2 . 8 8 )}$ & NA & NA \\
RP2.2 vs. RP1.1 & 0.48 & $0.74(0.32-1.71)$ & NA & NA \\
RP1.1 vs. RP1.2/RP2.2 & 0.78 & $1.12(0.50-2.53)$ & NA & NA \\
RP1.2 vs. RP1.1/RP2.2 & 0.06 & $1.63(0.98-2.72)$ & NA & NA \\
RP2.2 vs. RP1.1/RP1.2 & 0.06 & $0.62(038-1.01)$ & NA & NA \\
RP1 vs. RP2 & 0.09 & $1.39(0.95-2.05)$ & & \\
\hline
\end{tabular}

$H$, Caucasian $\left(n=4^{a}\right)$

\begin{tabular}{|c|c|c|c|c|}
\hline RP1.1 vs. RP1.2 & 0.74 & $0.94(0.67-1.33)$ & $24 \%$ & 0.26 \\
\hline RP1.2 vs. RP2.2 & 0.03 & 1.38 (1.04-1.83) & $1 \%$ & 0.39 \\
\hline RP2.2 vs. RP1.1 & 0.05 & $0.61(0.37-1.01)$ & $49 \%$ & 0.12 \\
\hline RP1.1 vs. RP1.2/RP2.2 & 0.64 & $1.08(0.78-1.50)$ & $40 \%$ & 0.17 \\
\hline RP1.2 vs. RP1.1/RP2.2 & 0.06 & $1.26(0.99-1.59)$ & $0 \%$ & 0.63 \\
\hline RP2.2 vs. RP1.1/RP1.2 & 0.01 & $0.71(0.54-0.93)$ & $37 \%$ & 0.19 \\
\hline RP1 vs. RP2 & 0.13 & $1.30(0.92-1.82)$ & $66 \%$ & 0.03 \\
\hline
\end{tabular}

I, Asian $\left(n=6^{a}\right)$

\begin{tabular}{lcccc}
\hline RP1.1 vs. RP1.2 & 0.41 & $1.17(0.81-1.71)$ & $72 \%$ & 0.003 \\
RP1.2 vs. RP2.2 & 0.14 & $1.46(0.88-2.42)$ & $0 \%$ & 0.98 \\
RP2.2 vs. RP1.1 & 0.05 & $0.62(0.38-1.01)$ & $0 \%$ & 0.48 \\
RP1.1 vs. RP1.2/RP2.2 & 0.31 & $1.22(0.83-1.81)$ & $76 \%$ & 0.0009 \\
RP1.2 vs. RP1.2/RP2.2 & 0.46 & $0.87(0.61-1.25)$ & $0 \%$ & 0.006 \\
RP2.2 vs. RP1.1/RP1.2 & 0.07 & $0.64(0.40-1.04)$ & $76 \%$ & 0.0008 \\
RP1 vs. RP2 & 0.23 & $1.23(0.88-1.74)$ & 0.67 \\
\hline
\end{tabular}

${ }^{a}$ Number of articles. Significant associations are denoted in bold font. Alleles of two and three repeats were designated as RP1 and RP2, respectively. Genotypes were designated as RP1.1=RP1/RP1, RP1.2=RP1/RP2 and RP2.2=RP2/RP2. RP, repeat; OR, odds ratio; CI, confidence interval; NA, not applicable.

IL-4 $(32,43)$. In addition, the rs79071878 polymorphism may also affect the transcription activity of IL-4 (54). However, despite the identifications of these potential mechanisms, the results concerning the association of IL-4 gene polymorphisms and cancer risk remain controversial. Thus, in order to clarify this association, a meta-analysis was performed in the present study to estimate the correlation between IL-4 gene polymorphisms (rs2243250, rs2070874 and rs79071878) and cancer susceptibility.

For IL-4 rs2243250 polymorphism, the present data suggested that this polymorphism was significantly associated with cancer risk. In subgroup analyses by cancer type, 
rs2243250 was demonstrated to be associated with a higher risk of gastric cancer and breast cancer. The CT/TT genotype carriers were at a lower risk of developing gastric cancer or breast cancer compared with individuals with the $\mathrm{CC}$ genotype. Furthermore, the CT genotype was demonstrated to be associated with an increased risk of prostate cancer compared with the CC/TT genotypes. These results suggested that this polymorphism may serve different roles in different types of malignancies. Further subgroup analysis by ethnicity revealed that the IL-4 rs2243250 polymorphism was correlated with an increased cancer risk in both Asian and Caucasian populations. The overall analysis for the IL-4 rs2070874 polymorphism yielded no significant association with general cancer risk. In the cancer-type subgroup analysis, a significant association of IL-4 rs2070874 polymorphism with leukemia and oral carcinoma was identified, with patients carrying the CT genotype or the $\mathrm{C}$ allele being more likely to develop oral carcinoma. By contrast, for leukemia the CT genotype carriers were at a lower risk of developing leukemia. It is worth noting that these results should be interpreted with caution, since our estimations regarding leukemia and oral carcinoma were based on one single study. Additionally, in ethnicity sub-analysis, the results indicated a significant association with cancer susceptibility among Asian populations under the recessive genetic model. Finally, the IL-4 rs79071878 polymorphism was overtly associated with a higher risk of cancer under the allelic model. The results of subgroup analyses indicated that IL-4 rs79071878 polymorphism was significantly associated with bladder cancer and breast cancer in certain genetic models, and an association between IL-4 rs79071878 polymorphism and cancer susceptibility was only observed among Caucasians, but not Asians. Overall, from general and subgroup analyses, it can be concluded that IL-4 gene polymorphisms may be important in the pathogenesis of certain types of cancer, and their effects on cancer risk may be ethnic specific. Nevertheless, the amount of relevant studies is not sufficient to draw a safe conclusion, and further well-designed studies with larger patient sample size will be required in the future to validate the present results.

Heterogeneity is one of the most important issues when performing meta-analysis. In the present meta-analysis, heterogeneity between studies existed in almost all comparisons. Therefore, we attempted to detect the source of heterogeneity by dividing included studies into different subgroups according to cancer type and ethnicity. The heterogeneity was drastically decreased in most subgroups, suggesting that these two factors contribute to a significant portion of heterogeneity in the present meta-analysis.

When interpreting the results of the present meta-analysis, several limitations should be considered. Firstly, the numbers of relevant studies were limited, and studies regarding several particular types of cancer were extremely lacking. Secondly, although funnel plots did not reveal any publication bias, the possibility of publication bias cannot be completely eliminated, since only published studies were included. Thirdly, the present results were based on unadjusted estimates, while a more precise analysis should have been adjusted by other factors, including smoking, age, and environmental factors. Finally, the present analyses did not consider the possibility of gene-gene or SNP-SNP interactions or the possibility of linkage disequilibrium between polymorphisms. Taking all these limitations into consideration, the results reported by the current study should be interpreted with caution.

In summary, the present results suggest that the IL-4 rs2243250 and rs79071878 polymorphisms were associated with cancer susceptibility. Further subgroup analyses revealed that the effects of IL-4 gene polymorphisms on cancer risk may vary depending on the cancer type and the ethnicity. However, given that the present results were based on limited number of case-control studies, further multi-center studies with larger sample size from different populations are warranted to confirm our results.

\section{References}

1. Ferlay J, Soerjomataram I, Dikshit R, Eser S, Mathers C, Rebelo M, Parkin DM, Forman D and Bray F: Cancer incidence and mortality worldwide: Sources, methods and major patterns in GLOBOCAN 2012. Int J Cancer 136: E359-E386, 2015.

2. Knox SS: From 'omics' to complex disease: A systems biology approach to gene-environment interactions in cancer. Cancer Cell Int 10: 11, 2010.

3. Jang JP, Baek IC, Choi EJ and Kim TG: Multiplex genotyping of cytokine gene SNPs using fluorescence bead array. PLoS One 10: e0118008, 2015.

4. Balkwill $\mathrm{F}$ and Mantovani A: Inflammation and cancer: Back to Virchow? Lancet 357: 539-545, 2001.

5. Leibovici D, Grossman HB, Dinney CP, Millikan RE, Lerner S, Wang Y, Gu J, Dong Q and Wu X: Polymorphisms in inflammation genes and bladder cancer: From initiation to recurrence, progression, and survival. J Clin Oncol 23: 5746-5756, 2005.

6. Baniyash M: Chronic inflammation, immunosuppression and cancer: New insights and outlook. Semin Cancer Biol 16: 80-88, 2006.

7. Aggarwal BB, Vijayalekshmi RV and Sung B: Targeting inflammatory pathways for prevention and therapy of cancer: Short-term friend, long-term foe. Clin Cancer Res 15: 425-430, 2009.

8. Bidwell J, Keen L, Gallagher G, Kimberly R, Huizinga T, McDermott MF, Oksenberg J, McNicholl J, Pociot F, Hardt C and D'Alfonso S: Cytokine gene polymorphism in human disease: On-line databases, supplement 1. Genes Immun 2: 61-70, 2001.

9. Wang HW and Joyce JA: Alternative activation of tumor-associated macrophages by IL-4: Priming for protumoral functions. Cell Cycle 9: 4824-4835, 2010.

10. Stang A: Critical evaluation of the Newcastle-Ottawa scale for the assessment of the quality of nonrandomized studies in meta-analyses. Eur J Epidemiol 25: 603-605, 2010.

11. Crusius JB, Canzian F, Capellá G, Peña AS, Pera G, Sala N, Agudo A, Rico F, Del Giudice G, Palli D, et al: Cytokine gene polymorphisms and the risk of adenocarcinoma of the stomach in the European prospective investigation into cancer and nutrition (EPIC-EURGAST). Ann Oncol 19: 1894-1902, 2008.

12. El-Omar EM, Rabkin CS, Gammon MD, Vaughan TL, Risch HA, Schoenberg JB, Stanford JL, Mayne ST, Goedert J, Blot WJ, et al: Increased risk of noncardia gastric cancer associated with proinflammatory cytokine gene polymorphisms. Gastroenterology 124: 1193-1201, 2003.

13. García-González MA, Lanas A, Quintero E, Nicolás D, Parra-Blanco A, Strunk M, Benito R, Angel Simón M, Santolaria S, Sopeña F, et al: Gastric cancer susceptibility is not linked to pro-and anti-inflammatory cytokine gene polymorphisms in whites: A Nationwide Multicenter Study in Spain. Am J Gastroenterol 102: 1878-1892, 2007.

14. Lai KC, Chen WC, Jeng LB, Li SY, Chou MC and Tsai FJ: Association of genetic polymorphisms of MK, IL-4, p16, p21, p53 genes and human gastric cancer in Taiwan. Eur J Surg Oncol 31: 1135-1140, 2005.

15. Pan XF, Wen Y, Loh M, Wen YY, Yang SJ, Zhao ZM, Tian Z, Huang H, Lan H, Chen F, et al: Interleukin- 4 and -8 gene polymorphisms and risk of gastric cancer in a population in Southwestern China. Asian Pac J Cancer Prev 15: 2951-2957, 2014.

16. Zambon CF, Basso D, Marchet A,Fasolo M,Stranges A,Schiavon S, Navaglia F, Greco E, Fogar P, Falda A, et al: IL-4-588C $>$ T polymorphism and IL-4 receptor alpha [Ex5+14A $>$ G; Ex11+828A $>$ G] haplotype concur in selecting $\mathrm{H}$. pylori cagA subtype infections. Clin Chim Acta 389: 139-145, 2008. 
17. Liang XL, Hu HB, Jia AP and Liu ZP: Association of PTPN11 and interleukin-4 gene polymorphisms and helicobacter pylori infection with susceptibility to gastric cancer. Hai Nan Yi Xue 2010: 1-5, 2010 (In Chinese).

18. Chu H, Wang M, Yan F, Zhong D, Shi D, Ma L, Pan X, Qin C, Yin $\mathrm{C}$ and Zhang Z: Polymorphisms in the IL-13 and IL-4R genes are associated with the development of renal cell carcinoma. Ann Oncol 23: 2114-2121, 2012

19. Cozar JM, Romero JM, Aptsiauri N, Vazquez F, Vilchez JR, Tallada M, Garrido F and Ruiz-Cabello F: High incidence of CTLA-4 AA (CT60) polymorphism in renal cell cancer. Hum Immunol 68: 698-704, 2007.

20. Joshi NN, Bhat S, Hake S, Kale M and Kannan S: Opposing effects of pro- and anti-inflammatory cytokine gene polymorphisms on the risk for breast cancer in western Indian women: A pilot study. Int J Immunogenet 41: 242-249, 2014.

21. Schonfeld SJ, Bhatti P, Brown EE, Linet MS, Simon SL, Weinstock RM, Hutchinson AA, Stovall M, Preston DL, Alexander $\mathrm{BH}$, et al: Polymorphisms in oxidative stress and inflammation pathway genes, low-dose ionizing radiation, and the risk of breast cancer among US radiologic technologists. Cancer Causes Control 21: 1857-1866, 2010.

22. Chen J, Ying XM, Huang XM, Huang P and Yan SC: Association between polymorphisms in selected inflammatory response genes and the risk of prostate cancer. Onco Targets Ther 9: 223-229, 2016.

23. Olson SH, Orlow I, Simon J, Tommasi D, Roy P, Bayuga S, Ludwig E, Zauber AG and Kurtz RC: Allergies, variants in IL-4 and IL-4R alpha genes, and risk of pancreatic cancer. Cancer Detect Prev 31: 345-351, 2007.

24. Welsh MM, Karagas MR, Kuriger JK, Houseman A, Spencer SK, Perry AE and Nelson HH: Genetic determinants of UV-susceptibility in non-melanoma skin cancer. PLoS One 6 : e20019, 2011

25. Howell WM, Turner SJ, Theaker JM and Bateman AC: Cytokine gene single nucleotide polymorphisms and susceptibility to and prognosis in cutaneous malignant melanoma. Eur J Immunogenet 30: 409-414, 2003.

26. Andrie E, Michos A, Kalampoki V, Pourtsidis A, Moschovi M, Polychronopoulou S, Athanasiadou-Piperopoulou F, Kalmanti M, Hatzakis A, Paraskevis D, et al: Genetic variants in immunoregulatory genes and risk for childhood lymphomas. Eur J Haematol 83: 334-342, 2009.

27. Monroy CM, Cortes AC, Lopez MS, D'Amelio AM Jr, Etzel CJ, Younes A, Strom SS and El-Zein RA: Hodgkin disease risk: Role of genetic polymorphisms and gene-gene interactions in inflammation pathway genes. Mol Carcinog 50: 36-46, 2011

28. Gaur P, Mittal M, Mohanti B and Das S: Functional variants of IL4 and IL6 genes and risk of tobacco-related oral carcinoma in high-risk Asian Indians. Oral Dis 17: 720-726, 2011

29. Tsai MH, Chen WC, Tsai CH, Hang LW and Tsai FJ: Interleukin-4 gene, but not the interleukin-1 beta gene polymorphism, is associated with oral cancer. J Clin Lab Anal 19: 93-98, 2005.

30. Vairaktaris E, Yapijakis C, Serefoglou Z, Avgoustidis D, Critselis E, Spyridonidou S, Vylliotis A, Derka S, Vassiliou S, Nkenke E and Patsouris E: Gene expression polymorphisms of interleukins-1 beta, $-4,-6,-8,-10$, and tumor necrosis factors-alpha, -beta: Regression analysis of their effect upon oral squamous cell carcinoma. J Cancer Res Clin Oncol 134: 821-832, 2008.

31. Lu Y, Wu Z, Peng Q, Ma L, Zhang X, Zhao J, Qin X and Li S: Role of IL-4 gene polymorphisms in HBV-related hepatocellular carcinoma in a Chinese population. PLoS One 9: e110061, 2014.

32. Saxena R, Chawla YK, Verma I and Kaur J: Effect of IL-12B, IL-2, TGF- $\beta 1$, and IL-4 polymorphism and expression on hepatitis B progression. J Interferon Cytokine Res 34: 117-128, 2014.

33. Li X, Shi W, Yu G, Lin L, Yang B, Li J, Guo W, Tang C, Wang H, $\mathrm{Gao} \mathrm{H}$, et al: Interleukin-4 -590T/C polymorphism influences the susceptibility to nonsmall cell lung cancer. DNA Cell Biol 31: 797-800, 2012

34. Gu J, Shen Y and Zhang Y: Association between interleukin-4 polymorphisms and environment and nonsmall cell lung cancer in Chinese population. J Cancer Res Ther 10 (Suppl): C135-C139, 2014.

35. Chang WS, Wang SC, Chuang CL, Ji HX, Hsiao CL, Hsu CM, Tsai CW, Liu SP, Hsu PC, Lo YL and Bau DT: Contribution of interleukin-4 genotypes to lung cancer risk in Taiwan. Anticancer Res 35: 6297-6301, 2015
36. Suchy J, Kłujszo-Grabowska E, Kładny J, Cybulski C, Wokołorczyk D, Szymańska-Pasternak J, Kurzawski G, Scott RJ and Lubiński J: Inflammatory response gene polymorphisms and their relationship with colorectal cancer risk. BMC Cancer 8: $112,2008$.

37. Wilkening S, Tavelin B, Canzian F, Enquist K, Palmqvist R, Altieri A, Hallmans G, Hemminki K, Lenner P and Försti A: Interleukin promoter polymorphisms and prognosis in colorectal cancer. Carcinogenesis 29: 1202-1206, 2008.

38. Yannopoulos A, Nikiteas N, Chatzitheofylaktou A and Tsigris C: The $(-590 \mathrm{C} / \mathrm{T})$ polymorphism in the interleukin-4 gene is associated with increased risk for early stages of corolectal adenocarcinoma. In Vivo 21: 1031-1035, 2007.

39. Wiemels JL, Wiencke JK, Kelsey KT, Moghadassi M, Rice T, Urayama KY, Miike R and Wrensch M: Allergy-related polymorphisms influence glioma status and serum IgE levels. Cancer Epidemiol Biomarkers Prev 16: 1229-1235, 2007.

40. Purdue MP, Sakoda LC, Graubard BI, Welch R, Chanock SJ, Sesterhenn IA, Rubertone MV, Erickson RL and McGlynn KA: A case-control investigation of immune function gene polymorphisms and risk of testicular germ cell tumors. Cancer Epidemiol Biomarkers Prev 16: 77-83, 2007.

41. Chu H, Ma L, Wang M, Shi D, Qin C, Yuan L, Yin C and Zhang Z: The polymorphisms of IL-4, IL-4R and IL-13 genes and bladder cancer risk in a Chinese population: A case-control study. Mol Biol Rep 39: 5349-5357, 2012.

42. Amirzargar AA, Bagheri M, Ghavamzadeh A, Alimoghadam K, Khosravi F, Rezaei N, Moheydin M, Ansaripour B, Moradi B and Nikbin B: Cytokine gene polymorphism in Iranian patients with chronic myelogenous leukaemia. Int J Immunogenet 32: 167-171, 2005.

43. Shamran HA, Hamza SJ, Yaseen NY, Al-Juboory AA, Taub DD, Price RL, Nagarkatti M, Nagarkatti PS and Singh UP: Impact of single nucleotide polymorphism in IL-4, IL-4R genes and systemic concentration of IL-4 on the incidence of glioma in Iraqi patients. Int J Med Sci 11: 1147-1153, 2014.

44. Lu Y: Association of variants of inflammation and immunity genes with susceptibility to gastric cancer. Nanjing Medical University, 2010 (In Chinese).

45. Gu F, Qureshi AA, Niu T, Kraft P, Guo Q, Hunter DJ and Han J: Interleukin and interleukin receptor gene polymorphisms and susceptibility to melanoma. Melanoma Res 18: 330-335, 2008.

46. Chen XL: TNF- $\beta$ IL-4 genes polymorphisms and Helicobacter pylori in gastric cancer and duodenal ulcer in the Chinese. Fujian Medical University, 2006 (In Chinese).

47. Tsai FJ, Chang CH, Chen CC, Hsia TC, Chen HY and Chen WC: Interleukin-4 gene intron-3 polymorphism is associated with transitional cell carcinoma of the urinary bladder. BJU Int 95: 432-435, 2005

48. Bozdoğan ST, Erol B, Dursun A, Bozdoğan G, Dönmez I, Mungan NA and Seydaoglu G: The IL-1RN and IL-4 gene polymorphisms are potential genetic markers of susceptibility to bladder cancer: A case-control study. World J Urol 33: 389-395, 2015.

49. Hsieh YY, Chang CC, Tsai CH, Lin CC and Tsai FJ: Interleukin (IL)-12 receptor betal codon $378 \mathrm{G}$ homozygote and allele, but not IL-1 (beta-511 promoter, 3953 exon 5, receptor antagonist), IL-2 114, IL-4-590 intron 3, IL-8 3'-UTR 2767, and IL-18 105 , are associated with higher susceptibility to leiomyoma. Fertil Steril 87: 886-895, 2007.

50. Shekari M, Kordi-Tamandani DM, MalekZadeh K, Sobti RC, Karimi S and Suri V: Effect of anti-inflammatory (IL-4, IL-10) cytokine genes in relation to risk of cervical carcinoma. Am J Clin Oncol 35: 514-519, 2012

51. Konwar R, Chaudhary P, Kumar S, Mishra D, Chattopadhyay N and Bid HK: Breast cancer risk associated with polymorphisms of IL-1RN and IL-4 gene in Indian women. Oncol Res 17: 367-372, 2009.

52. Kesarwani P, Ahirwar DK, Mandhani A and Mittal RD Association between-174 G/C promoter polymorphism of the interleukin-6 gene and progression of prostate cancer in North Indian population. DNA Cell Biol 27: 505-510, 2008.

53. Yang CM, Chen HC, Hou YY, Lee MC, Liou HH, Huang SJ, Yen LM, Eng DM, Hsieh YD and Ger LP: A high IL-4 production diplotype is associated with an increased risk but better prognosis of oral and pharyngeal carcinomas. Arch Oral Biol 59: 35-46, 2014.

54. Salimi S, Mohammadoo-Khorasani M, Yaghmaei M, Mokhtari M and Moossavi M: Possible association of IL-4 VNTR polymorphism with susceptibility to preeclampsia. Biomed Res Int 2014: 497031, 2014 